\title{
Fibered visible interferometry and adaptive optics: FRIEND at CHARA
}

\author{
M. A. Martinod ${ }^{1}$, D. Mourard ${ }^{1}$, P. Bério ${ }^{1}$, K. Perraut ${ }^{2}$, A. Meilland ${ }^{1}$, C. Bailet ${ }^{1}$, Y. Bresson ${ }^{1}$, T. ten Brummelaar ${ }^{3}$, \\ J. M. Clausse ${ }^{1}$, J. Dejonghe ${ }^{1}$, M. Ireland ${ }^{4}$, F. Millour ${ }^{1}$, J. D. Monnier ${ }^{5}$, J. Sturmann ${ }^{3}$, L. Sturmann ${ }^{3}$, and M. Tallon ${ }^{6}$ \\ ${ }^{1}$ Université Côte d'Azur, Observatoire de la Côte d'Azur, CNRS, Laboratoire Lagrange, France \\ e-mail: marc-antoine.martinod@oca.eu \\ 2 Université Grenoble Alpes, CNRS, IPAG, 38000 Grenoble, France \\ 3 CHARA Array, Mount Wilson Observatory, Mount Wilson, CA 91023, USA \\ ${ }^{4}$ Research School of Astronomy \& Astrophysics, Australian National University, Canberra, ACT 2611, Australia \\ 5 Department of Astronomy, University of Michigan, Ann Arbor, MI 48109, USA \\ ${ }^{6}$ Université de Lyon, Université Lyon 1, École Normale Supérieure de Lyon, CNRS, Centre de Recherche Astrophysique de Lyon \\ UMR5574, 69230 Saint-Genis-Laval, France
}

Received 16 June 2017 / Accepted 24 July 2018

\begin{abstract}
Aims. In the context of the future developments of long baseline interferometry at visible wavelengths, we have built a prototype instrument called Fibered spectrally Resolved Interferometer - New Design (FRIEND) based on single mode fibers and a new generation detector called Electron Multiplying Charge-Coupled Device (EMCCD). Installed on the Center for High Angular Resolution Astronomy (CHARA) array, it aims to estimate the performance of a fibered instrument in the visible when coupled with telescopes equipped with adaptive optics (AO) in partial correction.

Methods. We observed different sequences of targets and reference stars to study the compensation of the birefringence of the fibers, the coupling efficiency in various conditions of correction, and to calibrate our numerical model of signal-to-noise ratio (S/N). We also used a known binary star to demonstrate the reliability and the precision of our squared visibility and closure phase measurements. Results. We firstly present a reliable and stable solution for compensating the birefringence of the fibers with an improvement of a factor of 1.5 of the instrumental visibility. We then demonstrate an improvement by a factor of between 2.5 and 3 of the coupling efficiency when using the LABAO systems in closed loop. The third results of our paper is the demonstration of the correct calibration of the parameters of our $\mathrm{S} / \mathrm{N}$ estimator provided the correct excess noise factor of EMCCD is correctly taken into account. Finally with the measurements of the angular separation, difference of magnitude and individual diameters of the two components of $\zeta$ Ori A, we demonstrate the reliability and precision of our interferometric estimators, and in particular a median residual on the closure phase of $1.2^{\circ}$.
\end{abstract}

Key words. instrumentation: interferometers - instrumentation: high angular resolution - methods: observational techniques: interferometric

\section{Introduction}

In the last decade, the landscape of optical interferometry has radically changed. The Very Large Telescope Interferometer (VLTI; Glindemann et al. 2004) and the Center for High Angular Resolution Astronomy (CHARA; ten Brummelaar et al. 2005) arrays are operated as large open facilities, Magdalena Ridge Observatory Interferometer (MROI; Armstrong et al. 2016) is in a construction phase and Navy Precision Optical Interferometer (NPOI; Armstrong et al. 1998) is progressively evolving toward enhanced imaging capabilities. Adaptive optics (AO) is being installed on the small telescopes of the VLTI and of the CHARA array so as to correct the wavefront on the whole telescope pupil and push the sensitivity of their interferometric mode. Finally, new horizons are open with the recent operation of fringe tracking at kilohertz speed to compensate for real-time atmospheric piston variations and allow minute-long integration times on science targets (Gravity Collaboration 2017). These developments have already led to a rich harvest of scientific results, including first images of stellar surface (Roettenbacher et al. 2016; Ohnaka et al. 2017) and first large programs at high angular resolutions (Boyajian et al. 2012; Lazareff et al. 2017), bringing new constraints for stellar physics.

The determination of fundamental parameters of stars (radius, effective temperature, mass and age) is of paramount importance in Astrophysics, in particular to understand the properties and the formation of exoplanets, to improve our understanding of the physical processes in stars, and to study the structure and evolution of our Galaxy. The vast majority of stars have angular diameters of tenths to tens of milliseconds of arc, well beyond the diffraction limit of the largest telescopes including the future Extremely Large Telescope (ELT). Only long-baseline optical interferometry can provide an accurate angular diameter of these stars and allow us to derive relationships useful in extending our knowledge to a larger number of stars, at distances too far to accurately resolve their sizes. Using visible wavelengths is a good way to increase the angular resolution without longer baselines. Three visible instruments are in operation today: the Visible Imaging System for Interferometric Observations instrument at NPOI (NPOI/VISION), a 6T fibered combiner working with small apertures (Garcia et al. 2016), the Precision Astronomical Visible Observation 
instrument at CHARA (CHARA/PAVO), a 3T pupil-plane combiner with low spectral resolution (Ireland et al. 2008), and the Visible spEctroGraph and polArimeter instrument at CHARA (CHARA/VEGA), a 4T multimode combiner with medium and high spectral resolution (Mourard et al. 2009).

To go further, visible long-baseline interferometry is now implementing single mode guided optics for taking benefit of spatial filtering. Combined with adaptive optics and fast and low noise detectors, it opens very interesting possibilities. This scheme is used for a long time in the infrared (e.g., Astronomical Multi-BEam combineR (AMBER; Weigelt et al. 2016), Precision Integrated-Optics Near-infrared Imaging ExpeRiment (PIONIER; Wittkowski et al. 2017), General Relativity Analysis via Vlt InTerferometrY (GRAVITY; Gravity Collaboration 2017). A first attempt of single mode interferometry in the visible was performed with IOTA/SMART (Monnier et al. 2003) but it had to deal with huge injection fluctuations. NPOI/VISION is the first fibered visible combiner to be used, with six apertures of $12 \mathrm{~cm}$ which do not require AO systems. Our group in Nice has recently developed a prototype called Fibered spectrally Resolved Interferometer - New Design (FRIEND; Berio et al. 2014; Martinod et al. 2016) installed at the CHARA Array. The arrival of AO on CHARA (ten Brummelaar et al. 2014; Che et al. 2014) and the well known limitations of VEGA (Mourard et al. 2012) due to the multimode regime and the photon counting Intensified Charge-Coupled Device (ICCD) have driven the conceptual design of FRIEND. This prototype aims at studying the potential of using electron multiplying chargecoupled devices (EMCCD) and of coupling single mode optical fibers after the adaptive optics correction on the CHARA telescopes, and at demonstrating actual on-sky performance. It prepares the development of a future six-telescope visible instrument for the CHARA Array, called Stellar Parameters and Images with a Cophased Array (SPICA; Mourard et al. 2017).

The paper is organised as follows: in Sect. 2, we present the instrument, the estimators, and the data processing. We also define our estimators of squared visibility and closure phase. In Sect. 3, we present the results of the birefringence compensator that permits to optimize the transfer function of the instrument. The Sect. 4 details the results obtained on the limiting magnitude and coupling efficiency with single mode fibers and partial correction by adaptive optics. In Sect. 5, we present a study of the signal-to-noise ratio $(\mathrm{S} / \mathrm{N})$ on squared visibility measurements with fibers and an EMCCD, and in Sect. 6 we demonstrate the reliability of our $V^{2}$ and closure phase (CP hereafter) estimations on a known binary system, $\zeta$ Ori. Finally in Sect. 7 we conclude and discuss the impact of these results on a future 6T visible combiner for the CHARA Array.

\section{Presentation of FRIEND instrument}

FRIEND is a fibered three-telescope combiner operating in the $R$ band. It is installed on the VEGA tables (Mourard et al. 2009) to benefit from the existing modules and sources allowing the co-alignment and cophasing with the CHARA beams. FRIEND is equipped with a fast and low noise EMCCD OCAM2 detector (Gach et al. 2011) and uses Gaussian polarization-maintaining single mode optical fibers. Photometric channels permit to calibrate the contrast of the dispersed fringes.

\subsection{Optical layout}

A scheme of the FRIEND prototype is displayed in Fig. 1. The beams coming from the three telescopes or from the internal

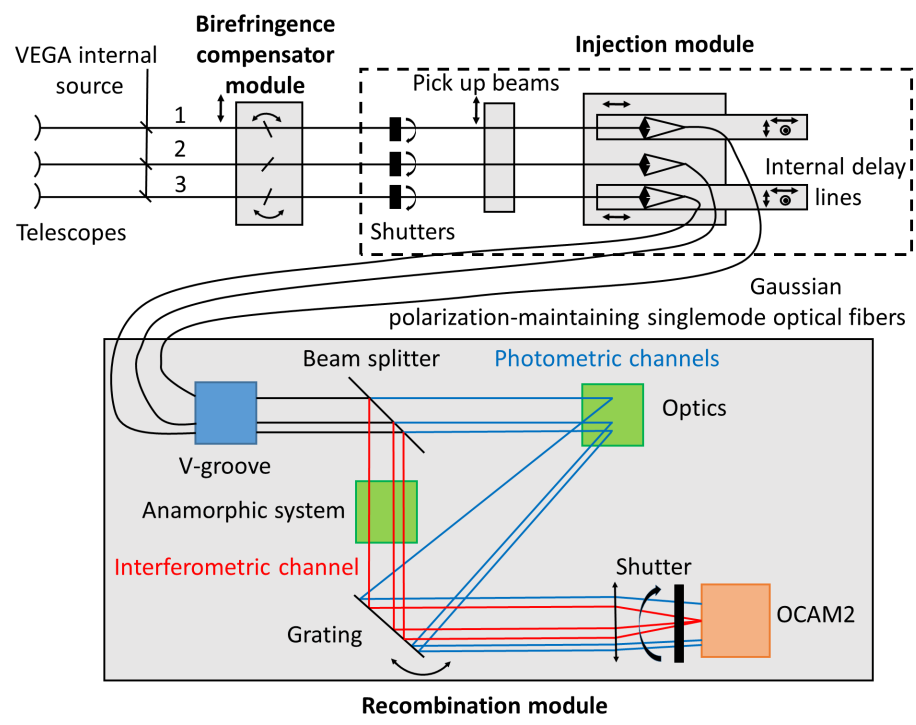

Fig. 1. Schematic presentation of FRIEND with the input beams, the birefringence compensator, the injection module, the beamsplitter that permits to extract the three photometric channels, the anamorphic system in the interferometric channel, and the spectrograph for all the channels.

source of VEGA go first through the birefringence compensator module. It aims at compensating the effect of the birefringence of the fibers in order to maximize the instrumental visibility (see Sect. 3).

Then the beams are redirected by the pick-up optics toward the injection module, where they are injected into Gaussian polarization-maintaining single mode optical fibers. The fibers have been chosen for a spectral bandwidth of $620-850 \mathrm{~nm}^{1}$. The fibers of beams 1 and 3 are mounted on internal delay lines used to compensate the internal delays and to cophase the FRIEND and VEGA instruments. The outputs of the fibers are rearranged on a $\mathrm{V}$-groove to encode the fringes on three different spatial frequencies: $3 D / \lambda$ for Base 1 (beams 2 and 3), $6 D / \lambda$ for Base 2 (beams 1 and 2), and $9 D / \lambda$ for Base 3 (beams 1 and 3), with $D$ the diameter of the collimated beams after the V-groove.

The fiber outputs are collimated and directed toward a 70/30 beam splitter separating the interferometric and the photometric channels. In the interferometric channel, the spatial sampling is adapted through an anamorphic system whereas the three photometric channels are oriented toward different places of the detector. Two different gratings offer a low (resp. medium) spectral resolution of $R=200$ (resp. $R=2400$ ) over the bandwidth $620-750 \mathrm{~nm}$. A mirror could be used in replacement of the grating for alignment purposes. Finally, the beams are imaged on the OCAM2 detector (Fig. 2).

\subsection{Main characteristics of the detector}

The detector is the OCAM2 EMCCD from First Light Imaging $^{2}$. It is a high-speed and low-noise detector able to run up to $1500 \mathrm{fps}$ with subelectron readout noise $\left(\sigma_{\mathrm{RON}}=\right.$ $0.14 \mathrm{e}^{-} \mathrm{px}^{-1} \mathrm{fr}^{-1}$; Feautrier et al. 2011). OCAM2 uses a custom CCD $(240 \times 240$ pixels $)$ developed by e2v technologies whose dark and Clock Induced Charge (CIC) signal is $N_{\mathrm{d}+\mathrm{c}}=$

\footnotetext{
1 http://wWw.nufern.com/pam/optical_fibers/960/ PM630-HP

2 https://www . first-light.fr/
} 


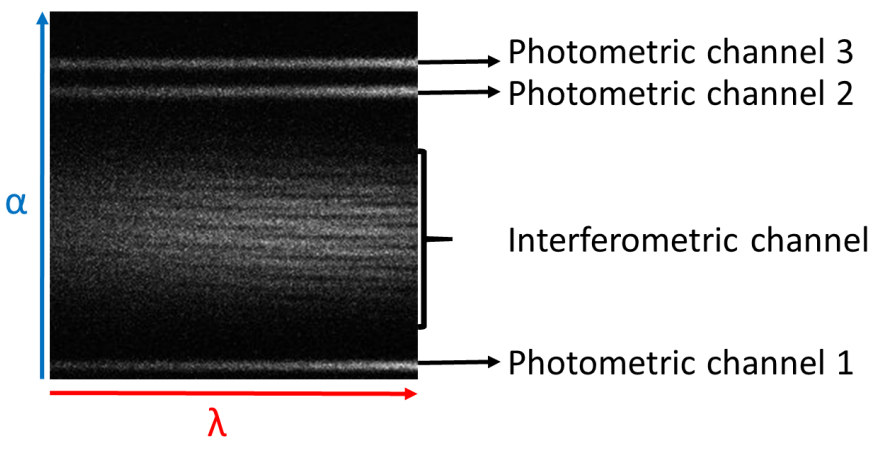

Fig. 2. Signal projected on the detector, obtained with the internal whitelight source of VEGA. From top to bottom: photometric channels 3 and 2, interferometric channel, photometric channel 1. The channels are spectrally dispersed along the horizontal axis and their spatial position are set along the vertical one.

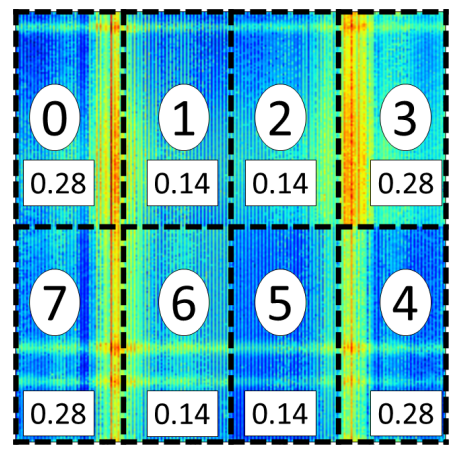

Fig. 3. Eight subareas of the pixel matrix of OCAM2. The numbers inside rectangular labels are the readout noise of the subarea in $\mathrm{e}^{-\mathrm{px}^{-1} \mathrm{fr}^{-1}}$.

$4.4 \mathrm{e}^{-} \mathrm{px}^{-1} \mathrm{~s}^{-1}$ when cooled at $-45^{\circ} \mathrm{C}$. Because of the moderate cooling, CIC and dark current are not distinguished in the OCAM2.

The amplification of the signal can be described as a stochastic process multiplying the variance of the input signal by an excess noise factor $F^{2}$ :

$\sigma_{\text {out }}^{2}=F^{2} G^{2} \sigma_{\text {in }}^{2}$,

with $\sigma_{\text {out }}^{2}$ the variance of the amplified signal, $\sigma_{\text {in }}^{2}$ the variance of the input signal in the amplification register and $G$ the amplification gain. It has been shown by Robbins \& Hadwen (2003) that $F^{2}=2$ at high gain.

In the following, the expression of the variance of the photon noise will integrate these characteristics and, after normalization, is written as

$\sigma_{\text {mod }}^{2}=F^{2}\left(N_{\mathrm{ph}}+N_{\mathrm{d}+\mathrm{c}}\right)$.

The matrix of pixels is divided in eight subareas of $60 \times 120$ pixels with their own amplification and readout registers (Fig. 3). Therefore, each subarea has its own effective amplification gain. In our detector, the readout noise of the side subareas is twice higher than in the central zones. Consequently, we decided to only use the four central subareas in the data processing. Our observations are made at a frame rate of $50 \mathrm{fps}$.

\subsection{Data processing}

The FRIEND data correspond to a classical three-telescope dispersed interferogram + three dispersed photometric channels.
Because of limited $\mathrm{S} / \mathrm{N}$ in the current installation of FRIEND, no differential visibilities are calculated and our data product is limited to squared visibility $\left(V^{2}\right)$ and CP estimation. In Appendix A, we present the details of the data flow. We focus here on the definition of the estimator of the interferometric observables.

\subsection{1. $V^{2}$ and $\sigma_{V^{2}}$}

The estimator of the squared visibility is given by Martinod et al. (2016):

$\left\langle\left|V_{i j}\right|^{2}\right\rangle=\frac{\left\langle\widetilde{E_{i j}}\right\rangle}{\int_{\Delta \lambda} \kappa_{i}(\lambda) \kappa_{j}(\lambda) \int_{\Delta \alpha}\left\langle P_{i}(\lambda, \alpha) P_{j}(\lambda, \alpha)\right\rangle \mathrm{d} \alpha \mathrm{d} \lambda}$

where denotes a quantity in the Fourier space, \langle\rangle denotes a temporal averaging over a series of frames. $\Delta \lambda$ and $\Delta \alpha$ are the spectral band and the spatial width of the photometric channel. $\left\langle\widetilde{E_{i j}}\right\rangle$ is the average, over the frames, of the unbiased power spectrum (PS hereafter) integrated over the support of the fringe signal of beams $i, j . P_{i}(\lambda, \alpha)$ is the flux of the beam $i$ in the interferogram at position $(\lambda, \alpha)$, using the information obtained in Step 2 of the data flow presented in Appendix A.

The uncertainty $\sigma_{V^{2}}$ on the measurement is estimated as

$\sigma_{V^{2}}=\sqrt{\sigma_{E_{i j}}^{2}\left(\frac{\partial V^{2}}{\partial E_{i j}}\right)^{2}+\sigma_{D}^{2}\left(\frac{\partial V^{2}}{\partial D}\right)^{2}}$

where $D$ corresponds to the denominator of Eq. (3).

To estimate $\sigma_{D}$, we first express $D$ as

$D=\int_{\Delta \lambda} \kappa_{i}(\lambda) \kappa_{j}(\lambda) \int_{\Delta \alpha} G_{i}(\lambda, \alpha) G_{j}(\lambda, \alpha)\left\langle p_{i} p_{j}\right\rangle \mathrm{d} \alpha \mathrm{d} \lambda$

with $p_{i}$ the total flux in the photometric channel $i$ and $G_{i}(\lambda, \alpha)$ the Gaussian pattern of the beam $i$ in the interferogram. We neglect the uncertainty on the pattern determination and thus, $\sigma_{D}$ could be expressed as

$\sigma_{D}^{2}=\sigma_{p_{i} p_{j}}^{2} \times\left\langle\left[\int_{\Delta \lambda} \kappa_{i}(\lambda) \kappa_{j}(\lambda) \int_{\Delta \alpha} G_{i}(\lambda, \alpha) G_{j}(\lambda, \alpha) \mathrm{d} \alpha \mathrm{d} \lambda\right]^{2}\right\rangle$

with $\sigma_{p_{i} p_{j}}^{2}=\left\langle\left(p_{i} p_{j}\right)^{2}\right\rangle-\left\langle\left(p_{i} p_{j}\right)\right\rangle^{2}$, the variance of the product of the total flux of beams $i$ and $j$.

We deduced the standard deviation of $\left\langle\widetilde{E_{i j}}\right\rangle$ from the variance of the top of the fringe-peak given by Gordon \& Buscher (2012):

$\sigma_{E_{i j}}=\sqrt{\text { PhotonNoise }+ \text { ReadNoise }+ \text { CoupledTerms }} \times \sqrt{\frac{N_{\mathrm{sz}}}{N_{\mathrm{f}}}}$

with $N_{\mathrm{f}}$ the number of frames, $N_{\mathrm{sz}}=9 \times 9$ pixels the number of pixels of the fringe-peak support.

Gordon \& Buscher (2012) give the expressions of PhotonNoise, ReadNoise, and CoupledTerms for photon and readout noises only. As previously said, we use our modified expression of the variance of the photon noise, as written in Eq. (2), and obtain

PhotonNoise $=2 \sigma_{\text {mod }}^{2}\left(\frac{N_{\text {ph }} V_{\text {instr }} V_{\text {star }}}{N_{\text {tel }}}\right)^{2} \widetilde{\mathrm{Gab}_{i j}}+\left(\sigma_{\text {mod }}^{2}\right)^{2}$

ReadNoise $=N_{\text {pix }} \sigma_{\text {RON }}^{2}+\left(N_{\text {pix }} \sigma_{\text {RON }}^{2}\right)^{2}$ 
CoupledTerms $=2 N_{\text {pix }} \sigma_{\text {RON }}^{2}\left(\frac{N_{\text {ph }} V_{\text {instr }} V_{\text {star }}}{N_{\text {tel }}}\right)^{2} \widetilde{\mathrm{Gab}_{i j}}$

$$
+2 \sigma_{\text {mod }}^{2} N_{\text {pix }} \sigma_{\text {RON }}^{2}
$$

where:

- $N_{\text {ph }}$ is the number of photons in the interferometric channel,

- $V_{\text {instr }}$ : the instrumental visibility,

- $V_{\text {star }}$ : the target visibility,

$-N_{\text {tel }}=3$ : the number of telescopes,

$\widetilde{\mathrm{Gab}_{i j}}$ is the shape factor. Its expression (see Appendix B for the demonstration) is given by

$\widetilde{\mathrm{Gab}_{i j}}=\frac{3}{\sqrt{\pi}}$.

The number of photon $N_{\mathrm{ph}}$ for one frame is defined by

$N_{\mathrm{ph}}=T_{\mathrm{C}} T_{\mathrm{OA}} T_{\mathrm{F}} \rho S_{\text {tel }} N_{\text {tel }} \Delta \lambda \mathrm{DIT} 10^{-0.4 m_{\mathrm{R}}} \Phi_{0} \mathrm{QE}$

where:

- $T_{\mathrm{C}}=0.017:$ the transmission of CHARA from the telescopes to the instrument (see Sect. 4.1),

$-T_{\mathrm{F}}=0.35:$ the transmission of the beam splitter and the grating,

- $T_{\mathrm{OA}}=0.765:$ the transmission of the adaptive optics devices,

- $\rho$ : the coupling efficiency,

$-S_{\text {tel }}=0.75 \mathrm{~m}^{2}:$ the effective collecting area of the telescope,

$-\Delta \lambda=60 \mathrm{~nm}$ : the spectral band,

- DIT $=0.02 \mathrm{~s}$ : the detector integration time,

- $m_{\mathrm{R}}$ : the magnitude of the target,

- $\Phi_{0}=6.2 \times 10^{7} \mathrm{ph} \mathrm{m}^{2} \mathrm{~nm}^{-1} \mathrm{~s}^{-1}$ : the reference flux at $m=0$,

- $\mathrm{QE}=0.9$ : the quantum efficiency of the detector.

$\sigma_{\text {mod }}^{2}$ and $\left(\frac{N_{\mathrm{ph}} V_{\text {instr }} V_{\text {star }}}{N_{\text {tel }}}\right)^{2} \widetilde{\mathrm{Gab}_{i j}}$ can be directly measured:

$-\sigma_{\text {mod }}^{2}=\overline{\mathrm{PS}_{1, \text { bias }}}$, it is the bias of the PS of the interferogram (Fig. A.2, upper-left) before processing performed in Eq. (A.2),

- $\left(\frac{N_{\mathrm{ph}} V_{\text {instr }} V_{\mathrm{star}}}{N_{\mathrm{tel}}}\right)^{2} \widetilde{\mathrm{Gab}_{i j}}=E_{i j}$, it is the energy of the fringe-peak

So Eqs. (8) and (10) can be rewritten as

PhotonNoise $=2 \overline{\mathrm{PS}_{1, \text { bias }}} E_{i j}+{\overline{\mathrm{PS}_{1, \text { bias }}}}^{2}$,

CoupledTerms $=2 N_{\text {pix }} \sigma_{\text {RON }}^{2} E_{i j}+2 \overline{\mathrm{PS}_{1, \text { bias }}} N_{\text {pix }} \sigma_{\text {RON }}^{2}$.

With these expressions, we now have a reliable numerical calculator of the uncertainty on the squared visibility.

\subsection{2. $\mathrm{CP}$ and $\sigma_{\mathrm{CP}}$}

The bispectrum estimator used in the data reduction software of FRIEND (Basden \& Haniff 2004) is given by

$B_{0, i j k}=B_{1, i j k}-\alpha\left(\left|C_{i j}\right|^{2}+\left|C_{j k}\right|^{2}+\left|C_{i k}\right|^{2}\right)+\beta N$

where $B_{0, i j k}$ and $B_{1, i j k}$ are the corrected and the raw bispectrum of telescopes $i, j, k,\left|C_{i j}\right|^{2}$ is the PS of the fringes $i j, N$ is the average flux of photons on the interferometric channel. Basden \& Haniff (2004) assume that only the real part is biased by the PS of the fringes and by the flux in the interferometric channel. $\alpha$ and $\beta$ are corrective coefficients which depend on the noise statistics and regime flux. In our processing, the coefficient $\alpha$ is estimated by the minimization of the variance of the corrected bispectrum outside the region of signal. The coefficient $\beta$

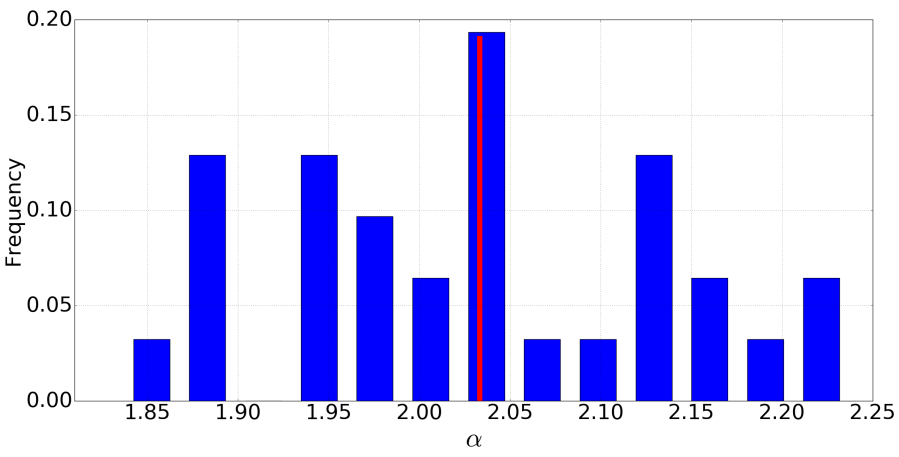

Fig. 4. Normalized histogram of the value of $\alpha$ parameter used in the computation of the unbiased bispectrum, as estimated on the data of the night 2017 Oct 14 (Table 4). The red line represents the mean value of this distribution.

is then set to have a null mean value of the average corrected bispectrum outside the region of signal. In Fig. 4, we plot the histogram of our estimation of $\alpha$ from the different sequences of observations of the night 2017 Oct 14 . We found $\alpha=2.0 \pm 0.1$ which is in perfect agreement with the value $\alpha=2$ proposed by Basden \& Haniff (2004) in the case of the use of a EMCCD without any threshold of the signal.

The closure phase $\mathrm{CP}$ can be computed as

$\mathrm{CP}(u, v)=\arctan \left(\frac{\sum \mathfrak{J}\left(\left\langle B_{0, i j k}\right\rangle\right)}{\sum \mathfrak{R}\left(\left\langle B_{0, i j k}\right\rangle\right)}\right)$

with $\sum \mathfrak{J}\left(\left\langle B_{0, i j k}\right\rangle\right)\left(\right.$ resp. $\left.\sum \mathfrak{R}\left(\left\langle B_{0, i j k}\right\rangle\right)\right)$ the sum of a $3 \times 3$ pixels area of the imaginary part (wrt real part) of the corrected bispectrum centered on the maximum of its modulus. $(u, v)$ are the temporal average co-ordinates of the third fringe-peak in the $(u, v)$ plan.

Tatulli \& Chelli (2005) give an estimator of the closure phase error for a centro-symmetrical object assuming small errors on phases of the pairs of telescopes and a noise model based on shot and readout noises only

$\sigma_{\Delta \phi}^{2}=\sigma_{\text {phot }}^{2}+\sigma_{\mathrm{RON}}^{2}$.

$\sigma_{\text {phot }}^{2}$ and $\sigma_{\text {RON }}^{2}$ are the contributions of the photon noise and readout noise in the variance of the bispectrum. For the first contribution, the expression of Tatulli \& Chelli (2005) is rewritten to take into account the modified expression of the variance of the photon noise (Eq. (2)) and is now presented as (see Appendix C for more details):

$$
\begin{aligned}
\sigma_{\mathrm{phot}}^{2} & =\frac{N_{\text {tel }}^{3}}{2\left|V_{12} V_{23} V_{13}\right|^{2}}\left(\frac{N_{\text {tel }}^{3}}{N_{\mathrm{ph}}^{6}}\right)\left(\sigma_{\mathrm{mod}}^{2}\right)^{3}-\frac{1}{\left|V_{12} V_{23} V_{13}\right|}\left(\frac{N_{\text {tel }}}{N_{\mathrm{ph}}}\right)^{3} \\
& +\frac{N_{\text {tel }}^{2}\left(\left|V_{12}\right|^{2}+\left|V_{23}\right|^{2}+\left|V_{13}\right|^{2}\right)}{2\left|V_{12} V_{23} V_{13}\right|^{2}}\left(\frac{N_{\text {tel }}^{2}}{N_{\mathrm{ph}}^{4}}\right)\left(\sigma_{\mathrm{mod}}^{2}\right)^{2} \\
& -\frac{\left|V_{12}\right|^{4}+\left|V_{23}\right|^{4}+\left|V_{13}\right|^{4}}{2\left|V_{12} V_{23} V_{13}\right|^{2}}\left(\frac{N_{\text {tel }}}{N_{\mathrm{ph}}}\right)^{2} \\
& +\frac{\left|V_{12}\right|^{2}\left|V_{23}\right|^{2}+\left|V_{23}\right|^{2}\left|V_{13}\right|^{2}+\left|V_{12}\right|^{2}\left|V_{13}\right|^{2}}{\left|V_{12} V_{23} V_{13}\right|^{2}}\left(\frac{N_{\text {tel }}}{N_{\mathrm{ph}}}\right)^{2} \\
& +\frac{\left|V_{12}\right|^{2}\left|V_{23}\right|^{2}+\left|V_{23}\right|^{2}\left|V_{13}\right|^{2}+\left|V_{12}\right|^{2}\left|V_{13}\right|^{2}}{2\left|V_{12} V_{23} V_{13}\right|^{2}}\left(\frac{N_{\text {tel }}^{2}}{N_{\mathrm{ph}}^{2}}\right)\left(\sigma_{\text {mod }}^{2}\right) \\
& -\frac{\left|V_{12} V_{23} V_{13}\right|\left(\left|V_{12}\right|^{2}+\left|V_{23}\right|^{2}+\left|V_{13}\right|^{2}\right)}{\left|V_{12} V_{23} V_{13}\right|^{2}}\left(\frac{N_{\text {tel }}}{N_{\mathrm{ph}}}\right)
\end{aligned}
$$


$\left|V_{i j}\right|$ is the modulus of the visibility of beams $i$ and $j$. $\sigma_{\text {RON }}^{2}$ remains unchanged:

$$
\begin{aligned}
\sigma_{\mathrm{RON}}^{2}= & \frac{N_{\mathrm{pix}}^{3} \sigma_{\mathrm{RON}}^{6}+3 N_{\mathrm{pix}}^{2} \sigma_{\mathrm{RON}}^{6}}{2\left|V_{12} V_{23} V_{13}\right|^{2}}\left(\frac{N_{\text {tel }}}{N_{\mathrm{ph}}}\right)^{6} \\
& +\frac{\left(\left|V_{12}\right|^{2}+\left|V_{23}\right|^{2}+\left|V_{13}\right|^{2}\right)\left(3 N_{\mathrm{pix}} \sigma_{\mathrm{RON}}^{4}+N_{\mathrm{pix}}^{2} \sigma_{\mathrm{RON}}^{4}\right)}{2\left|V_{12} V_{23} V_{13}\right|^{2}}\left(\frac{N_{\text {tel }}}{N_{\mathrm{ph}}}\right)^{4} \\
& +\frac{\left(\left|V_{12}\right|^{2}\left|V_{23}\right|^{2}+\left|V_{23}\right|^{2}\left|V_{13}\right|^{2}+\left|V_{12}\right|^{2}\left|V_{13}\right|^{2}\right) N_{\mathrm{pix}} \sigma_{\mathrm{RON}}^{2}}{2\left|V_{12} V_{23} V_{13}\right|^{2}}\left(\frac{N_{\text {tel }}}{N_{\mathrm{ph}}}\right)^{2} .
\end{aligned}
$$

With these expressions, we now have an improved version of a numerical estimator of the variance of the closure phase measurements.

\section{Compensating for the birefringence of the fibers}

The birefringence of the fibers introduces optical path differences between the polarization state of the beams. Therefore the fringe patterns of the two polarizations are shifted, which generates a decrease of the instrumental visibility. We have adapted the VLTI/PIONIER solution (Lazareff et al. 2012) and used a tiltable plate of $\alpha$-Barium Borate ( $\alpha B B o$; the choice of this material is explained in Appendix D) to design the birefringence compensator. On beam 2, the plate is fixed at an incidence of $40^{\circ}$ to avoid ghost images and the tilts on beams 1 and 3 introduce optical path differences on the fringes 1-2 and 2-3 that permit to compensate for the internal polarized delays of the fibers. We developed a numerical model of the birefringence compensator in order to optimize its behavior and compared its results to the measurements performed in laboratory and on sky (Fig. 5).

In top panel of this figure, we present the measurements of the fringe contrast made on the internal source and their interpretation with our numerical model. The visibility presents a periodic modulation (period $\simeq 1^{\circ}$ ) defined by the thickness of the plate superimposed to a large scale envelop defined by the coherent length of the experience. The overall maximum of the fringe visibility is found for an angle of incidence of $\theta_{3}=24^{\circ}$. On-sky tests were made on the target $\gamma$ Cas with the telescopes W2 on beam 1, E2 on beam 2 and E1 on beam 3, during the night of 27 Sep 2016. We recorded fringe data with and without the system of compensation, and for different orientations of the plates 1 and 3 around the position found with the internal source. The results are presented in the bottom panel of Fig. 5 for the baseline E2E1 on beams 2 and 3. These results show, and this is also true on beams 1 and 2, that the optimum of the transfer function on the sky is the same than with the internal source. This also means that the external instrumental polarization on the considered CHARA Coudé trains is negligible. The small discrepancies between the on-sky and the internal measurements may be due to the variation of conditions on the sky: turbulence, quality of the fringe stabilisation, as the observations were spread over more than one hour.

The on-sky measurements were repeated at different hours, for different nights and different telescopes. It appears that it was never necessary to correct the position of the movable plates determined on the internal sources, meaning that no instrumental polarization of the CHARA Coudé trains has been detected in our observing conditions. The improvement by a factor of 1.5 of the instrumental squared visibility and the stability of the correction are thus demonstrated.
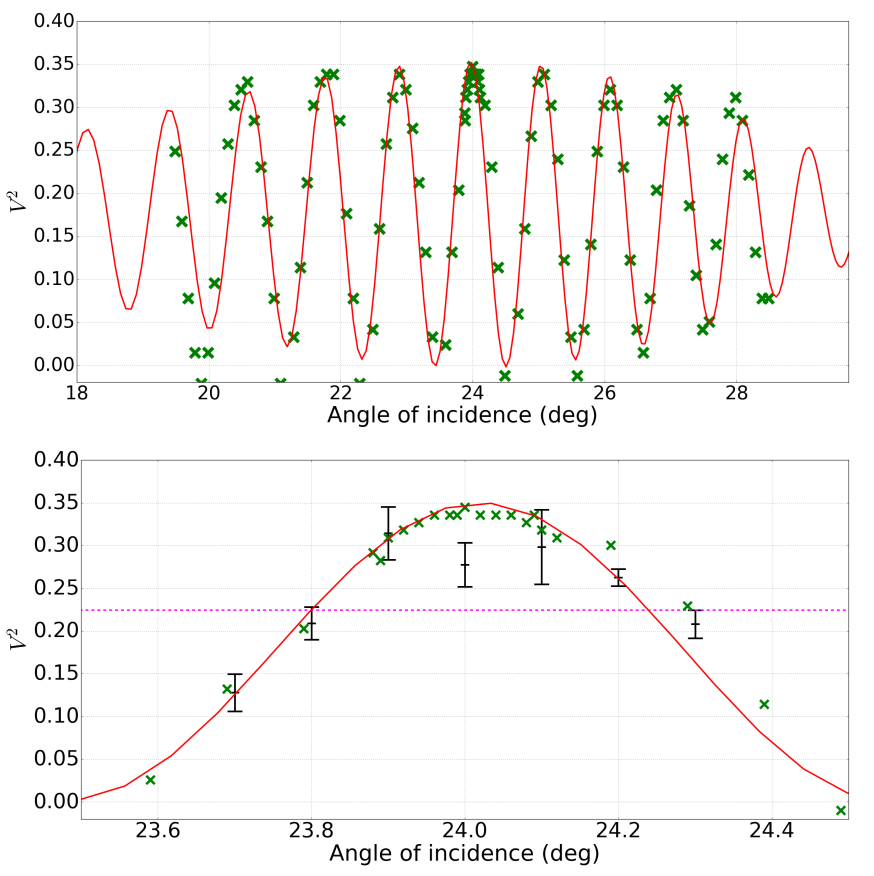

Fig. 5. Top panel: effect of the orientation of the plate 3 on the instrumental visibility of the fringe system $2-3$. The prediction by the numerical model (solid red line) is superimposed to the measurements (green crosses) made on the internal source. Bottom panel: magnification of the area around the optimal angular position of the plate. The on-sky measurements without the system of compensation of the birefringence (dashed magenta line) and with the system in place (black pluses) are plotted. Each black plus point and its error bar are respectively the average and standard deviation of a sample of five measurements from five blocks of 6000 frames.

\section{On-sky coupling efficiency}

The second important characteristics of a fibered instrument is the quality (level and stability) of the coupling efficiency. The fiber coupling efficiency has been studied for almost thirty years (Shaklan \& Roddier 1988; Ruilier \& Cassaing 2001; Meimon et al. 2013) but mainly in the case of good AO correction. The case of low AO correction has only been theoretically studied (Tatulli et al. 2010). So we need to characterize it in practice in order to secure the expected performance at this AO regime. The Strehl ratio of the CHARA AO systems (ten Brummelaar et al. 2014; Che et al. 2014) is expected, under good seeing conditions, to be around $80 \%$ in the $H$-band which may correspond to almost $25 \%$ at $700 \mathrm{~nm}$. This means a partial AO correction regime, which generates flux instabilities into a single-mode fiber and hence a temporally variable coupling efficiency that needs to be assessed. We were also looking for solutions to maximize the coupling efficiency under these conditions. We used FRIEND firstly without adaptive optics correction and more recently with the LABAO systems in operation.

\subsection{Transmission and coupling efficiency without $A O$ correction}

The stars observed during the night 2016 Sep 30 (see Table 1) range from magnitude 2.32 to 5.1. In Fig. 6, we plot, as a function of the magnitude, the mean flux over the night, measured in the interferogram and in each of the three photometric channels. 


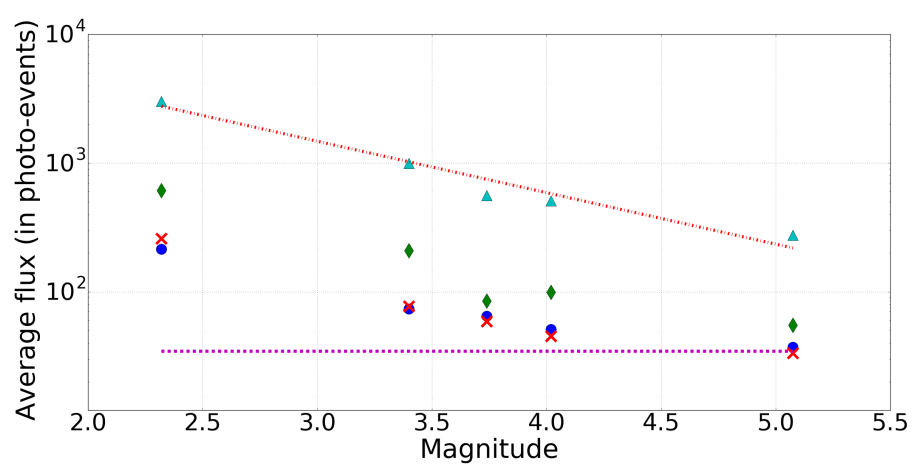

Fig. 6. Average flux in the interferometric channel (triangles) and in photometric channels (dots = beam 1 , diamonds = beam 2 , and crosses = beam 3 ) versus the magnitude $m_{\mathrm{R}}$. The mean level of the detector noise in a photometric channel (purple dashed line) and the photonmagnitude relation (red dash-dotted line), with the adjustment of the constant term in the exponent $\left(F=10^{-0.4 m_{\mathrm{R}}+4.37}\right)$, are also displayed.

Table 1. Magnitude ( $R$ band) of the stars used for the estimation of the transmission.

\begin{tabular}{cccccc}
\hline \hline Star & $\gamma$ Cas & HD 11415 & HD 3360 & HD 2905 & HD 3240 \\
\hline Magnitude & 2.32 & 3.4 & 3.74 & 4.02 & 5.1 \\
\hline
\end{tabular}

The mean $r_{0}$ for this measure is evaluated to $14 \mathrm{~cm}$ at $0.55 \mu \mathrm{m}$, corresponding for the $1 \mathrm{~m}$ CHARA telescopes to $N_{\mathrm{S}}=$ 31 speckles at $0.69 \mu \mathrm{m}$. Therefore the expected coupling efficiency, usually defined, for a pupil with a central obstruction of $25 \%$ of the pupil diameter (Ruilier 1998), as $0.69 \times$ SR with SR the Strehl ratio, is evaluated to $2.2 \%$. As a result of the fit of our flux measurement, we deduce a global transmission of $0.015 \%$. With the internal transmission of FRIEND of $35 \%$ in the interferometric channel, the $90 \%$ of quantum efficiency of the detector and the CHARA transmission of 1.7\% (CHARA meeting 2013, priv. comm.), we deduce a coupling efficiency of $2.8 \%$, close to the first estimation.

This low coupling efficiency without adaptive optics has a strong consequence on the photometric channels, receiving only $30 \%$ of the incoming flux and where frequent values under the noise level are measured. To avoid bias in the interferometric measurements, it is necessary to apply a filtering (see Sorting 2 in Appendix A) which consists in rejecting the frames where the flux of at least one of the photometric channel for a given baseline is below a certain threshold, defined as a multiplying factor to the average standard deviation of the integrated dark signal in the corresponding photometric channel. To avoid a too-large rejection of frames while preserving an unbiased estimation of the squared visibility, we set this factor to 1.5. This demonstrates that the effect of a poor coupling efficiency could be drastically amplified in terms of observing efficiency when combining many different beams.

\subsection{Coupling efficiency with the CHARA LABAO systems}

The CHARA LABAO systems (ten Brummelaar et al. 2014) are dedicated to the correction of the quasi-static aberrations of the six beams, at low frequency and using an internal beacon. They are composed of a deformable mirror with 37 actuators and a Shack-Hartmann wavefront sensor with a lenslet of 32 subapertures. On stars brighter than a magnitude of four in the visible, they can be used in closed loop at a frame rate of $40 \mathrm{~Hz}$.
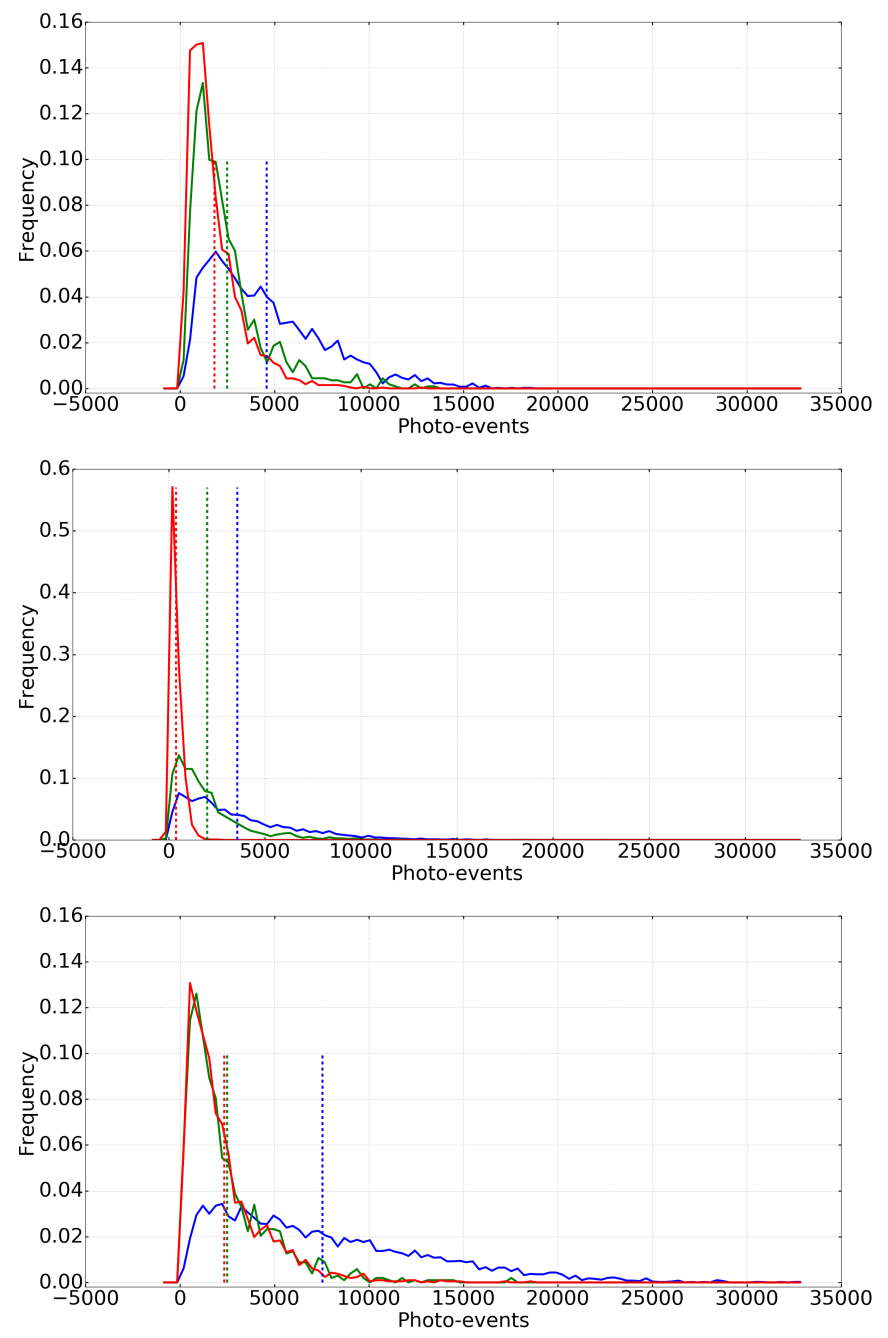

Fig. 7. Distribution of the injected photo-events per frame for S2 (top panel), S1 (middle panel), W2 (bottom panel) telescope. The blue (resp. green, red) solid line is the distribution of photon-events per frame in closed loop (resp. open loop, flatten) mode and the blue (resp. green, red) dashed line represents the corresponding average number of photoevents per frame.

Although this mode is not expected to provide a very good correction of the atmospheric turbulence (this is the goal of the second correction level described in Che et al. 2014), we used three of them with FRIEND during the night 2017 Oct 16, to study their influence on the coupling efficiency in the optical fibers. We tested three modes of the LABAO systems. The first one, which is the default one, is called "flatten" and corresponds to the best day-time alignment. The second mode is the closed loop with the $40 \mathrm{~Hz}$ loop in activity. The last one is the open loop, which is activated right after a closed loop and where the deformable mirror keeps its last shape. The tip-tilt is corrected by a dedicated system installed on the M2 mirror on each telescope of CHARA (Che et al. 2014).

We observed Capella $\left(m_{\mathrm{R}}=-0.52\right)$ with an average $r_{0}$ of $8 \mathrm{~cm}$ and processed the data to extract the photometry of each beam in the photometric channels of FRIEND. The distribution of photo-events per frame is computed for each mode and the results are presented in Fig. 7. In Table 2 we summarize the mean values for the three modes and include an estimation of the corresponding coupling efficiency using the results of Sect. 4.1. 
Table 2. Average value of photo-events per frame and corresponding coupling efficiency for the three modes of the LABAO.

\begin{tabular}{ccccccc}
\hline \hline \multirow{2}{*}{ Mode } & \multicolumn{3}{c}{ Photo-events } & \multicolumn{3}{c}{ Coupling efficiency (\%) } \\
\cline { 2 - 7 } & S2 & S1 & W2 & S2 & S1 & W2 \\
\hline Closed loop & 4577 & 3570 & 7544 & 2.99 & 2.12 & 3.8 \\
Open loop & 2502 & 2001 & 2497 & 1.63 & 1.19 & 1.26 \\
Flatten & 1819 & 373 & 2350 & 1.19 & 0.22 & 1.19 \\
\hline
\end{tabular}

The results obtained with telescope $\mathrm{S} 1$ are quite different from the other telescopes. This is mainly due to a poor alignment of the wavefront sensor at the time of our observations and thus a bad behavior of the interaction matrix for this beam. We noticed that the transmission differs from one telescope to another because of the different aging of the coating of the primary mirror. The estimation of the coupling efficiency takes this also into account. The main outcome of this observation is that it demonstrates that the closed loop mode of the LABAO on bright stars improves the coupling efficiency by a factor of between 2.5 and 3.2 in the visible, in other words, more than a magnitude.

\section{On-sky $\mathrm{S} / \mathrm{N}$ on $\mathrm{V}^{2}$}

The $\mathrm{S} / \mathrm{N}$ in the power spectrum of the interferogram has been already modeled by different authors (Gordon \& Buscher 2012; Mourard et al. 2017). As previously said, we use an improved model of the photon noise for an EMCCD, including dark current and CIC, by using the expression $\sigma_{\text {mod }}^{2}$ presented in Eq. (2), and considering the integral of the fringe-peak:

$S / N=\frac{\sqrt{N_{\mathrm{f}}}\left(\frac{N_{\mathrm{ph}} V_{\mathrm{instr}} V_{\mathrm{star}}}{N_{\mathrm{tel}}}\right)^{2} \widetilde{\mathrm{Gab}}_{i j}}{\sqrt{\text { PhotonNoise + ReadNoise + CoupledTerms } \sqrt{N_{\mathrm{sz}}}}}$,

whose quantities have already been defined by Eqs. (8), (9), (10), and (12).

The estimations from Eq. (20) have been compared with onsky measurements performed on the star HD 3360, during the night of 2016 Sep 30 with the telescopes E1, W2, and W1 on beams 1,2 , and 3 (Table 3 ). Figure 8 presents the measured $\mathrm{S} / \mathrm{N}$ as a function of the $V^{2}$ measurements made on the different baselines and for different times. We overplot the numerical estimations of the $\mathrm{S} / \mathrm{N}$ according to Eq. (20), and using the the mean Strehl ratio of $3.5 \%$ for the night (red solid curve). To estimate the uncertainty on these $\mathrm{S} / \mathrm{N}$ estimations, we also plot the estimations in the cases of the best Strehl ratio (4.3\%) and the worst one (2.6\%) for the night (black solid curve). This figure demonstrates that our numerical description matches very well with the actual on-sky measurements. It is important to note that not considering the amplification noise in the modeling leads to optimistic estimations of $\mathrm{S} / \mathrm{N}$ (green dashed curve in Fig. 8). We can note some outlier measurements for $V^{2}$ below 0.2. They correspond to the longest baseline (E1W1), where the fringe contrast is very low and for which we also accumulate the tracking errors of the two short baselines in the current setup of the group delay tracker.

\section{Zeta Ori A observations}

The binary system $\zeta$ Ori A (Hummel et al. 2013) has been observed with FRIEND with the S2, S1, and W2 telescopes in closed loop on the LABAO systems and with CLIMB as group

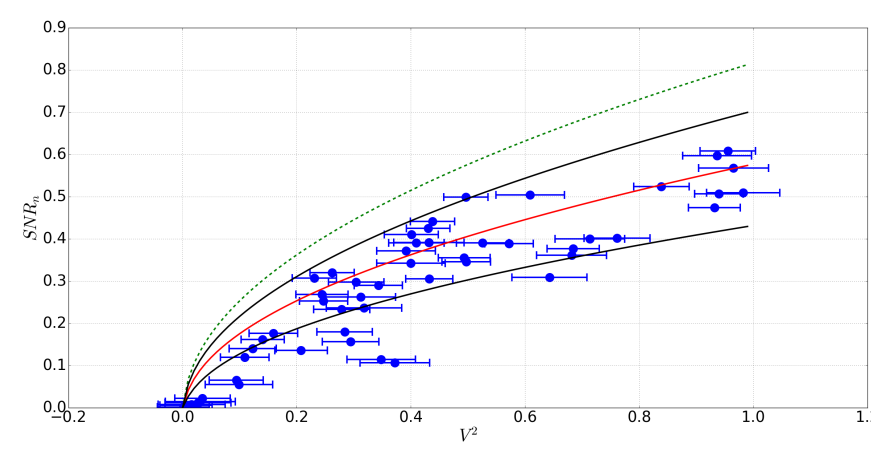

Fig. 8. Normalized $\mathrm{S} / \mathrm{N}$ of the $V^{2}$ measurements versus $V^{2}$ for the observation of HD 3360 of $2016 \mathrm{Sep} 30$ (blue dots) and our model of S/N estimation considering the mean Strehl ratio (3.5\%, red solid curve), the worst one (2.6\%, lower black solid curve), the best one (4.3\%, upper black solid curve) and without the amplification noise (green dashed curve). The last case is obtained with the mean Strehl ratio.

Table 3. Log of the observations in low spectral resolution mode.

\begin{tabular}{clllll}
\hline \hline \multirow{2}{*}{ Date } & \multirow{2}{*}{\begin{tabular}{l}
\multirow{2}{*}{ (h) } \\
2016 Sep 30
\end{tabular}} & Target & \multicolumn{2}{c}{ Baseline } & \multirow{2}{*}{$r_{0}$} \\
\cline { 4 - 5 } & 8h35 & HD 3360 & $108 / 222 / 314$ & $-2 / 36 / 24$ & 12 \\
& $9 \mathrm{~h} 13$ & HD 3360 & $222 / 106 / 312$ & $44 / 6 / 32$ & 13 \\
& $11 \mathrm{~h} 13$ & HD 3360 & $217 / 97 / 300$ & $70 / 32 / 59$ & 14 \\
& $12 \mathrm{~h} 33$ & HD 3360 & $216 / 89 / 292$ & $89 / 54 / 79$ & 15 \\
& $13 \mathrm{~h} 04$ & HD 3360 & $216 / 86 / 291$ & $-83 / 63 / 87$ & 16 \\
\hline
\end{tabular}

Notes. The date, time (UT), target name, projected baselines, and Fried parameter (at $0.55 \mu \mathrm{m}$ ) are given.

delay tracker (see Table 4). During the second night (2017 Oct 14), some instrumental issues prevented us from correctly calibrating the squared visibilities of the target and we consider only the closure phase estimations for that night. Each block of data (6000 frames of $20 \mathrm{~ms}$, i.e., two minutes of observation) gives an estimate of $V^{2}$ and CP for the science star or the reference star. The integration time of the detector is set to $20 \mathrm{~ms}$. For the typical conditions of the Mount Wilson observatory (coherence time of $10 \mathrm{~ms}$ ), it corresponds to the optimum value for the $\mathrm{S} / \mathrm{N}$ of the interferometric measurements, as stated by Buscher (1988) and validated on FRIEND by Martinod et al. (2016). The measurements on $\zeta$ Ori A are calibrated with those of the reference star $\kappa$ Ori which is a B05Ia star with $m_{\mathrm{R}}=2.09$. Its uniform-disk angular diameter in the $R$ band according to SearchCal version 5.0.1 is $\theta_{\mathrm{UD}, \mathrm{R}}=0.540 \pm 0.057$ mas.

\subsection{Instrumental visibility}

To estimate the instrumental squared visibility $V_{\mathrm{instr},}^{2}$, we used each raw measurements $V_{\text {raw,ref, } i}^{2}$ on the reference stars and a calculation of the squared visibility $V_{\text {th,ref, } i}^{2}$ based on their known angular diameters to compute the squared instrumental visibility $V_{\text {instr }, i}^{2}=V_{\text {raw,ref }, i}^{2} / V_{\text {th,ref, } i}^{2}$. For each night and each baseline, the two estimations of the instrumental visibility based on the reference star are linearly interpolated to obtain the value of the transfer function $V_{0, i}^{2}$ at the time of the observation of the science target. The calibrated measurements $V_{\mathrm{star}, i}^{2}$ on the targets could then be obtained as $V_{\mathrm{star}, i}^{2}=V_{\text {raw,target }, i}^{2} / V_{0, i}^{2}$.

The uncertainty on the diameter of the reference star and the dispersion of $V_{\text {raw,ref, } i}^{2}$ are taken into account when calculating 
Table 4. Log of the observations in low spectral resolution mode.

\begin{tabular}{ccclll}
\hline \hline \multirow{2}{*}{ Date } & \multirow{2}{*}{\begin{tabular}{c}
\multirow{2}{*}{ Th } \\
$(\mathrm{h})$
\end{tabular}} & & \multicolumn{2}{c}{ Target } & \multicolumn{2}{c}{ Baseline } & $r_{0}$ \\
\cline { 4 - 5 } & & $B_{\mathrm{p}}(\mathrm{m})$ & $P A\left(^{\circ}\right)$ & \\
\hline \multirow{2}{*}{2017 Oct 12 } & $10 \mathrm{~h} 25$ & $\kappa$ Ori & $25 / 117 / 143$ & $8.3 /-5.8 /-10$ & 9.8 \\
& $10 \mathrm{~h} 56$ & $\zeta$ Ori A & $28 / 137 / 164$ & $0.8 /-13 /-111$ & 12.7 \\
& $11 \mathrm{~h} 54$ & $\kappa$ Ori & $25 / 130 / 154$ & $-10 /-25 /-22$ & 10.8 \\
\multirow{2}{*}{2017 Oct 14 } & $10 \mathrm{~h} 06$ & $\kappa$ Ori & $25 / 116 / 141$ & $10 /-3.4 /-1$ & 7.7 \\
& $10 \mathrm{~h} 53$ & $\zeta$ Ori A & $28 / 137 / 164$ & $-0.1 /-14 /-12$ & 8.6 \\
& $11 \mathrm{~h} 12$ & $\zeta$ Ori A & $28 / 140 / 167$ & $-3.6 /-18 /-15$ & 8.1 \\
& $12 \mathrm{~h} 35$ & $\kappa$ Ori & $26 / 142 / 167$ & $-18 /-32 /-29$ & 7.2 \\
\hline
\end{tabular}

Notes. The date, time (UT), target name, projected baselines (resp. $\mathrm{S} 2 \mathrm{~S} 1, \mathrm{~S} 2 \mathrm{~W} 2$ and S1W2), and Fried parameter (at $0.55 \mu \mathrm{m}$ ) are given.

the uncertainty on the individual estimates $V_{\text {instr }, i}^{2}$. To correctly account for the systematics caused by potential non-calibrated sources of degradation of the signal coming either from the instrument or from the atmosphere, the uncertainty on $V_{0, i}^{2}$ is finally taken as the squared root of the sum of the average variance on the individual estimated $V_{\mathrm{instr}, i}^{2}$, and the variance of the residuals of the linear fit done to obtain $V_{0, i}^{2}$.

The raw squared visibilities of reference star and science star as well as the squared instrumental visibilities and the transfer function are displayed in Fig. 9. We chose to have a good sample of the variations of the closure phase and squared visibilities rather than optimizing the quality of the estimation of the transfer function by frequent switches between the target and the reference star. Therefore, the observing time on the science star is significantly longer than the individual observations on the reference star.

\subsection{Results}

We used the LITpro $^{3}$ tool (Tallon-Bosc et al. 2008) to adjust a binary model build with two uniform disks on the calibrated data ( $V^{2}$ and the closure phase measurements). The results are presented in Table 5 and in Fig. 10 for $V^{2}$ and Fig. 11 for CP. For the night of Oct 12, the data permitted to fit the whole set of parameters. However, due to the lack of reference star for the night of Oct 14, we were only able to adjust the position $x$ and $y$, and we set the values for the other parameters to the ones found for Oct 12 . We deduce a visual magnitude difference $\Delta m=2.4 \pm 0.1$ which is consistent with the value $\Delta m=2.2 \pm 0.1$ given by Hummel et al. (2013). We also deduce a separation $23.89 \pm 0.44$ mas (resp. $24.23 \pm 0.15$ mas) and a position angle of $81.42 \pm 0.33^{\circ}$ (resp. $81.00 \pm 0.15^{\circ}$ ) for the night of Oct 12 (resp. Oct 14).

The long baselines that we have used for these observations permit to correctly constrain the angular diameter of the primary star. This is obviously not so easy for the companion because of the difference of magnitude. Hummel et al. (2013) estimated the diameters of both stars which are, respectively $0.48 \pm 0.04$ mas (determined by intensity interferometry) and $0.18 \pm 0.05$, at $443 \mathrm{~nm}$. They deduced the last one from the magnitude difference with the strong hypothesis that both stars have the same effective temperature. It is estimated at $29500 \pm 1000 \mathrm{~K}$ (Bouret et al. 2008). Our estimation of Diameter 1 (0.54 \pm 0.01 at $690 \mathrm{~nm}$ ) matches the previous estimation within $1.5 \sigma$. Part of the difference could be explained by the difference of wavelengths in the two estimations. Concerning the companion $\mathrm{Ab}$,

\footnotetext{
3 http://www.jmmc.fr/litpro
}
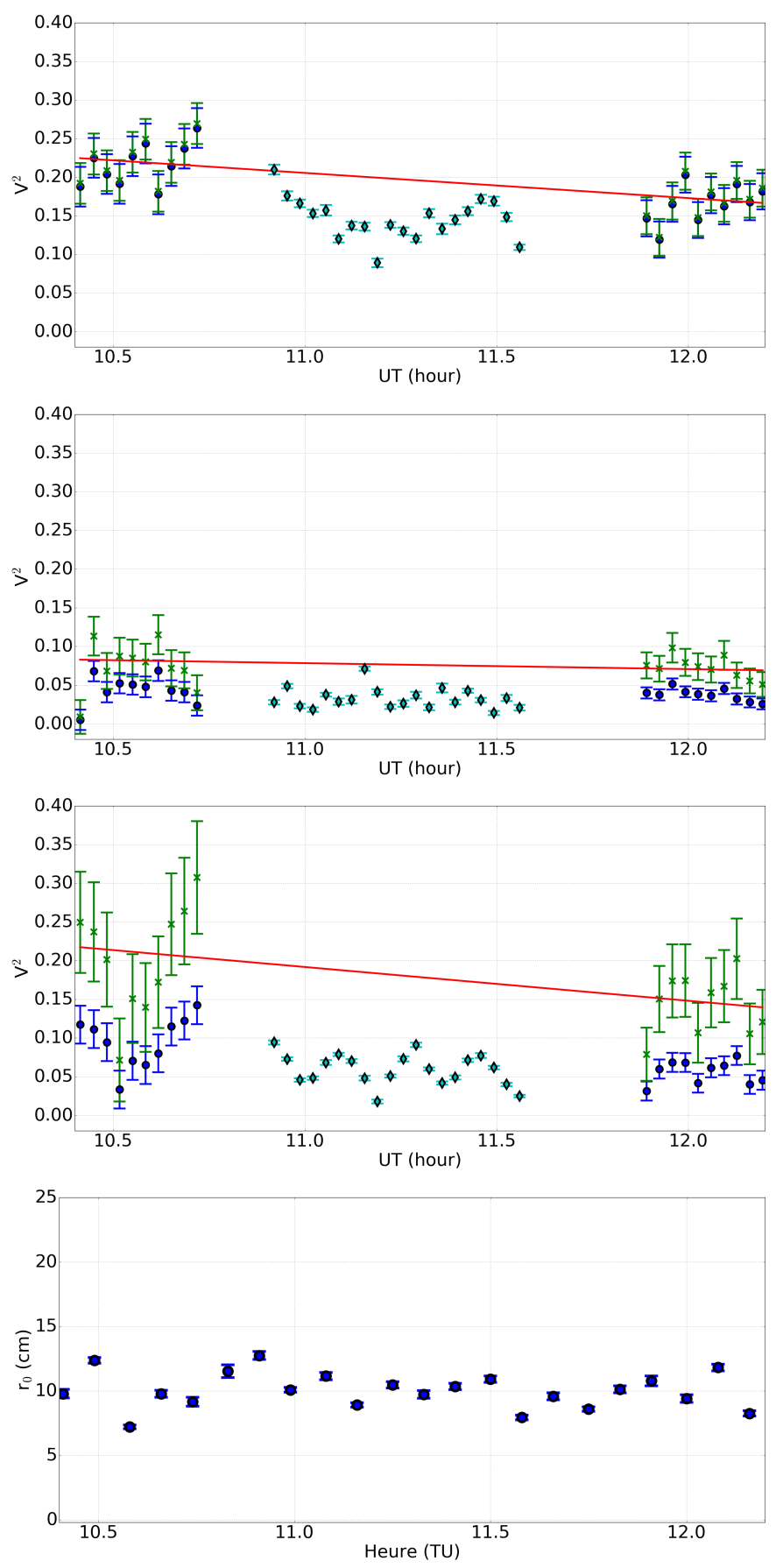

Fig. 9. Squared visibilities on $\zeta$ Ori A versus time during the night of 2017 Oct 12. From top to bottom panels: baselines S2S1, S2W2, and $\mathrm{S} 1 \mathrm{~W} 2$ for the reference star $\kappa$ Ori (blue dots), the target $\zeta$ Ori A (cyan diamonds), the squared instrumental visibilities $V_{\text {instr }, i}^{2}$ (green crosses), and the transfer function $V_{\text {instr }}^{2}$ obtained from a linear adjustment (red curve). The last plot is the evolution of the $r_{0}$ during the observation.

our angular diameter and its difference of flux with Aa lead to an estimation of the effective temperature $T_{\text {eff }}=15500 \pm 2600 \mathrm{~K}$. These parameters permit to revisit the spectral classification of $\zeta$ Ori $\mathrm{Ab}$ as B5II, instead of B1IV.

In Fig. 12, we plot the estimated and predicted positions of the companion around the primary star with the predicted orbit of Hummel et al. (2013). A Monte-Carlo simulation, based on the uncertainties of the orbital parameters, permitted to compute the error bars on the predicted positions. Furthermore, 
M. A. Martinod et al.: Optical interferometry at visible wavelengths with single mode fibers and adaptive optics
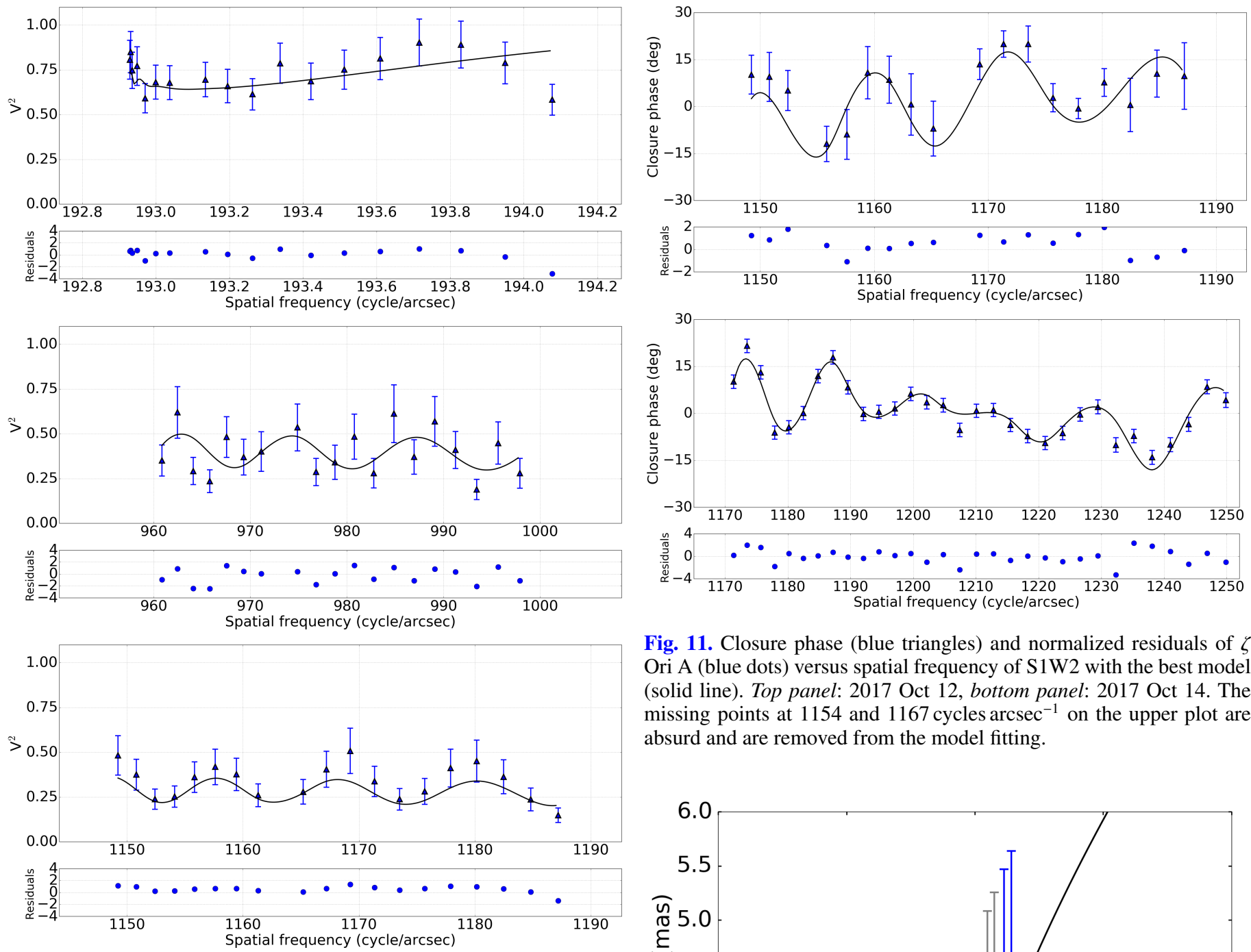

Fig. 10. Calibrated squared visibility (blue triangles) and normalized residuals of $\zeta$ Ori A (blue dots) versus spatial frequency for the night 2017 Oct 12 with the best model (solid line). From top to bottom panels: baselines S1S2, S2W2, and S1W2. The missing points on all baselines around 970 cycles $\operatorname{arcsec}^{-1}$ were absurd and they were removed from the plot and the model fitting.

Table 5. Parameters of the best model found for the two nights at $\lambda=$ $0.69 \mu \mathrm{m}$.

\begin{tabular}{ccc}
\hline \hline & 2017 Oct 12 & 2017 Oct 14 \\
\hline Diameter 1 (mas) & $0.54 \pm 0.01$ & \\
Diameter 2 (mas) & $0.45 \pm 0.12$ & \\
Flux ratio & $0.102 \pm 0.01$ & \\
$x$ & $23.6 \pm 0.45$ & $24.32 \pm 0.27$ \\
$y$ & $3.56 \pm 0.13$ & $3.88 \pm 0.11$ \\
Reduced $\chi^{2}$ & 1.14 & 1.59 \\
\hline
\end{tabular}

Notes. The correlation coefficients between Diameter 1, Diameter 2 and the flux ratio are lower than 0.5 .

our two positions were added in the fit of the orbital parameters and a better overall solution was found with a new period $P=2688.9$ days (instead of 2687.3 day) and a new epoch $T 0=2452735.8$ (instead of $T 0=2452734.2)$. The bottom plot

Fig. 11. Closure phase (blue triangles) and normalized residuals of $\zeta$ Ori A (blue dots) versus spatial frequency of S1W2 with the best model (solid line). Top panel: 2017 Oct 12, bottom panel: 2017 Oct 14. The missing points at 1154 and 1167 cycles arcsec $^{-1}$ on the upper plot are absurd and are removed from the model fitting.

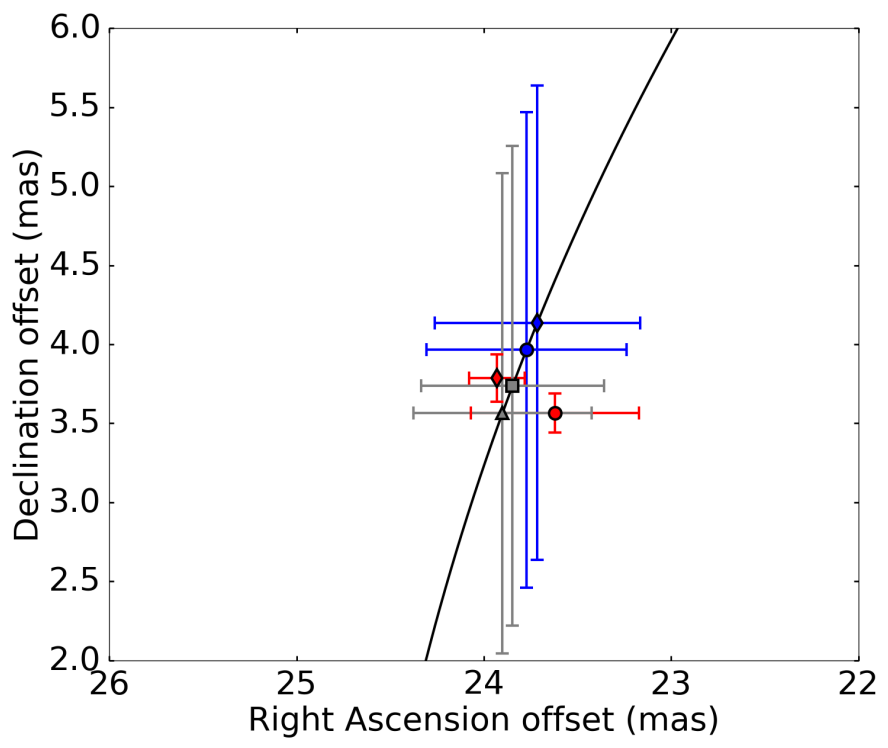

Fig. 12. Predicted orbit of the companion around the primary star (black curve) with the predicted positions from Hummel et al. (2013; blue markers), and the measurements (red markers). We plot positions on 2017 Oct 12 (circles) and on 2017 Oct 14 (diamonds). We also plot the predicted positions computed with the new orbital parameters including our measurements for 2017 Oct 12 (gray triangles) and 14 (gray squares).

of Fig. 12 used these new values to compute the orbit and the predicted positions for both nights and the agreement is even better.

These results on $\zeta$ Ori A clearly demonstrates the reliability and the precision of the FRIEND observations and permits to 
fully validate the instrument and its data flow. For the night 2017 Oct 14 , the average uncertainty on the closure phase estimations is $2.2^{\circ}$ and the median value of the residuals is $1.2^{\circ}$.

\section{Conclusion}

This paper presents recent progresses for fibered interferometry at visible wavelengths with partial adaptive optics correction. We successively demonstrate the quality and stability of the birefringence correction, and quantify the great improvement brought by adaptive optics for the coupling efficiency. We also demonstrate the reliability of our $\mathrm{S} / \mathrm{N}$ estimations and predictions provided that the so-called excess noise factor of EMCCD is correctly taken into account. Finally, our observations of the companion of $\zeta$ Ori A have permitted to demonstrate the reliability and precision of our estimators. These achievements are of strong interest to derive the performance of a future six-telescope visible combiner SPICA for the CHARA Array and to design a potential visible instrument for the VLTI (Millour et al. 2018).

The framework of adaptive optics developments for long baseline interferometric arrays opens very motivating opportunities for enhancing the performance at visible wavelengths, both in terms of sensitivity and precision. These activities will continue in the future thanks to new observations made using FRIEND and the second level of CHARA-AO with the goal of continuing the optimization of the coupling efficiency.

Acknowledgements. The FRIEND project is funded by the Institut Nationa des Sciences de l'Univers (CNRS/INSU), Observatoire de la Côte d'Azur, the LAGRANGE Laboratory and the European funding program FEDER. This work is based upon observations obtained with the Georgia State University Center for High Angular Resolution Astronomy Array at Mount Wilson Observatory. The CHARA Array is supported by the National Science Foundation under Grants No. AST-1211929 and AST-1411654. We warmly thank Christian Hummel (ESO) for the computation of the new orbital solution for $\zeta$ Ori A.

\section{References}

Armstrong, J. T., Mozurkewich, D., Rickard, L. J., et al. 1998, ApJ, 496, 550 Armstrong, J., Baines, E., \& Schmitt, H. 2016, in Proc. SPIE, 9907, 990702 Basden, A. G., \& Haniff, C. A. 2004, MNRAS, 347, 1187

Berio, P., Bresson, Y., \& Clausse, J. M. 2014, in Proc. SPIE, 9146, 914616
Bouret, J.-C., Donati, J.-F., Martins, F., et al. 2008, MNRAS, 389, 75 Boyajian, T. S., McAlister, H. A., van Belle, G., et al. 2012, ApJ, 746, 101

Buscher, D. 1988, MNRAS, 235, 1203

Che, X., Sturmann, L., \& Monnier, J. D. 2014, in Proc. SPIE, 9148, 914830

Feautrier, P., Gach, J.-L., Balard, P., et al. 2011, PASP, 123, 263

Gach, J.-L., Balard, P., Stadler, E., Guillaume, C., \& Feautrier, P. 2011, in Second International Conference on Adaptive Optics for Extremely Large Telescopes, 44, http://ao4elt2. lesia.obspm. fr

Garcia, E. V., Muterspaugh, M. W., van Belle, G., et al. 2016, PASP, 128, 055004 Glindemann, A., Albertsen, M., \& Andolfato, L. 2004, in Proc. SPIE, 5491, 447

Gordon, J. A., \& Buscher, D. F. 2012, A\&A, 541, A46

Gravity Collaboration (Abuter, R., et al.) 2017, A\&A, 602, A94

Hirsch, M., Wareham, R. J., Martin-Fernandez, M. L., Hobson, M. P., \& Rolfe, D. J. 2013, PLOS ONE, 8, 1

Hummel, C. A., Rivinius, T., Nieva, M.-F., et al. 2013, A\&A, 554, A52

Ireland, M. J., Mérand, A., \& ten Brummelaar, T. A. 2008, in Proc. SPIE, 7013, 701324

Koechlin, L., Lawson, P. R., Mourard, D., et al. 1996, Appl. Opt., 35, 3002

Lazareff, B., Le Bouquin, J., \& Berger, J. 2012, A\&A, 543, A31

Lazareff, B., Berger, J.-P., Kluska, J., et al. 2017, A\&A, 599, A85

Martinod, M., Berio, P., \& Mourard, D. 2016, in Proc. SPIE, 9907, 99071H

Meimon, S., Cassaing, F., \& Prévôt, G. 2013, J. Opt., 15, 035707

Millour, F., Ottogalli, S., Maamri, M., et al. 2018, Exp. Astron., submitted [arXiv:1808.09848]

Monnier, J. D., Berger, J. P., \& Millan-Gabet, R. 2003, in Interferometry for Optical Astronomy II, ed. W. A. Traub, Proc. SPIE, 4838, 1127

Mourard, D., Clausse, J. M., Marcotto, A., et al. 2009, A\&A, 508, 1073

Mourard, D., Challouf, M., \& Ligi, R. 2012, in Proc. SPIE, 8445, 84450K

Mourard, D., Bério, P., Perraut, K., et al. 2017, J. Opt. Soc. Am. A, 34, A37

Ohnaka, K., Weigelt, G., \& Hofmann, K.-H. 2017, Nature, 548, 310

Robbins, M. 2005, Internal Document of e2v Technologies

Robbins, M. S., \& Hadwen, B. J. 2003, IEEE Trans. Electron Devices, 50, 1227

Roettenbacher, R. M., Monnier, J. D., Korhonen, H., et al. 2016, Nature, 533, 217

Ruilier, C. 1998, Astron. Interferom., 3350, 319

Ruilier, C., \& Cassaing, F. 2001, J. Opt. Soc. Am. A, 18, 143

Shaklan, S., \& Roddier, F. 1988, Appl. Opt., 27, 2334

Tallon-Bosc, I., Tallon, M., \& Thiébaut, E. 2008, in Proc. SPIE, 7013, 70131J

Tatulli, E., \& Chelli, A. 2005, J. Opt. Soc. Am. A, 22, 1589

Tatulli, E., Blind, N., Berger, J. P., Chelli, A., \& Malbet, F. 2010, A\&A, 524, A65

ten Brummelaar, T. A., McAlister, H. A., Ridgway, S. T., et al. 2005, ApJ, 628, 453

ten Brummelaar, T., Che, X., \& McAlister, H. 2014, in Proc. SPIE, 9148, 91484Q

Weigelt, G., Hofmann, K.-H., Schertl, D., et al. 2016, A\&A, 594, A106

Wittkowski, M., Abellán, F. J., Arroyo-Torres, B., et al. 2017, A\&A, 606, L1 


\section{Appendix A: The FRIEND data flow}

During the observations, the science data are completed by auxiliary data required for correcting the cosmetic of the detector and for estimating the photometry of each beam in the interferogram. All data are recorded at a rate of 50 frames per second and we define a block as a series of 6000 single exposures $(2 \mathrm{mn}$ of time). The acquisition sequence is done as following:

A. Dark: Acquisition of ten blocks of dark (all shutters closed) to produce a map called the master dark and a table of gains called the master gain.

B. Shutters 1, 2, 3: Each internal shutter is opened to record successively the internal white-light source feeding fiber $i$ and seen at the same time in the photometric channel $i$ and in the interferometric channel. We obtained the required geometrical information (position and width of the photometric channels, shape of each beam in the interferogram) and the $\kappa_{i}(\lambda)$ coefficients (ratio of the flux of beam $i$ in the interferogram to the flux in the photometric channel $i$, for each wavelength).

C. Foreground data: The internal white-light source illuminates the detector with all shutters opened the internal delay lines placed out of coherence. We obtain the power spectrum (PS hereafter) of the photon noise.

D. Fringe data: On-sky acquisition to produce the squared visibility and the closure phase.

We have tested the stability of the different products of steps A, $\mathrm{B}$, and $\mathrm{C}$ and concluded that achieving these measurements once a day before the start of the night is sufficient.

The data flow of FRIEND is schematically presented in Fig. A.1. Each step of the processing produces intermediate products necessary for the next steps and for quality check.

Different frame selections are made all along the processing. With Sorting 1 we removed the frames with extreme values (maximum intensity of the image larger than three times the median of the maximum intensities) whereas Sorting 2 removes frames when one of the fluxes in the photometric channels ( 1 and 2 for fringes $1-2,2$ and 3 for fringes $2-3,1$ and 3 for fringes $1-3$, and 1,2 and 3 for closure phase) is below a certain threshold (see Sect. 4.1). Details of the different steps are described below:

Step 1: The dark frames are sorted for flashes and averaged to produce the "master dark". We also compute the histogram of the pixel intensities to estimate the "master gain", that is, the amplification gain of each of the subareas following the method described by Robbins (2005). This gives us all the required information for the preprocessing of the data frames

ppframe $=\frac{\text { frame }- \text { master dark }}{\text { master gain }} \times K$

where $K=19.12 \mathrm{e}^{-} \mathrm{ADU}^{-1}$ is the system gain. This equation is applied to all subsequent steps.

Step 2: The location and width of the photometric channels and of the interferogram are estimated by a double Gaussian fit on the integration of the shutter data. This gives us also the exact pattern of each beam in the interferogram. The $\kappa_{i}(\lambda)$ coefficients are finally calculated.

Step 3: The PS of the preprocessed dark frames is computed over the area of the interferogram. It is used later on to correct the PS of the fringe data. The threshold used for the photometric sorting is determined by computing the standard deviation of the intensity in the dark frames.

Step 4: The PS of the preprocessed foreground frames is computed over the area of the interferogram. It is used to remove the

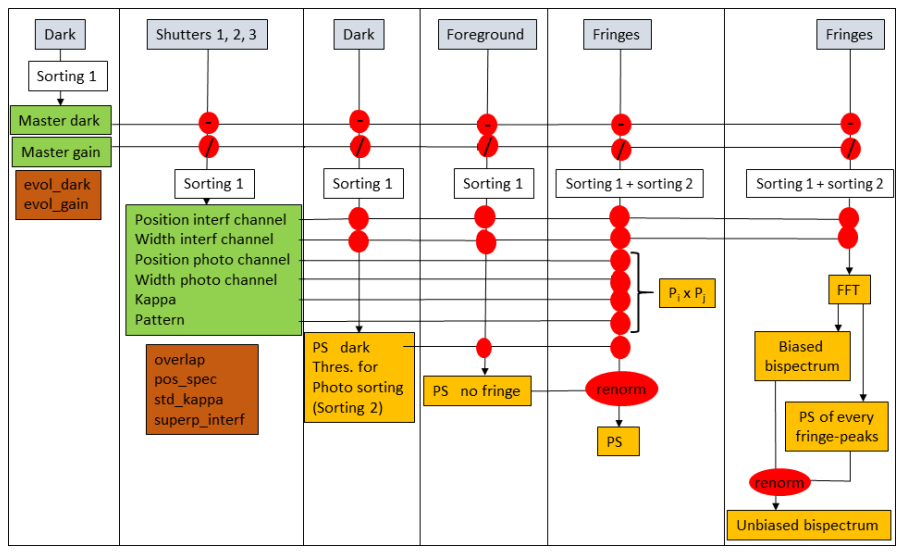

Fig. A.1. Data flow of FRIEND represented, from left to right, as the six steps explained in the text. There are the different raw data (grey boxes), the products of calibration (green boxes), the quality controls (brown boxes), and the products of the processing (orange boxes). Some products are required to proceed to the enxt step (red circles). Different selections are made on the raw frames (Sorting boxes): Sorting 1 removes the frames with flashes and Sorting 2 removes frames on photometric criteria.
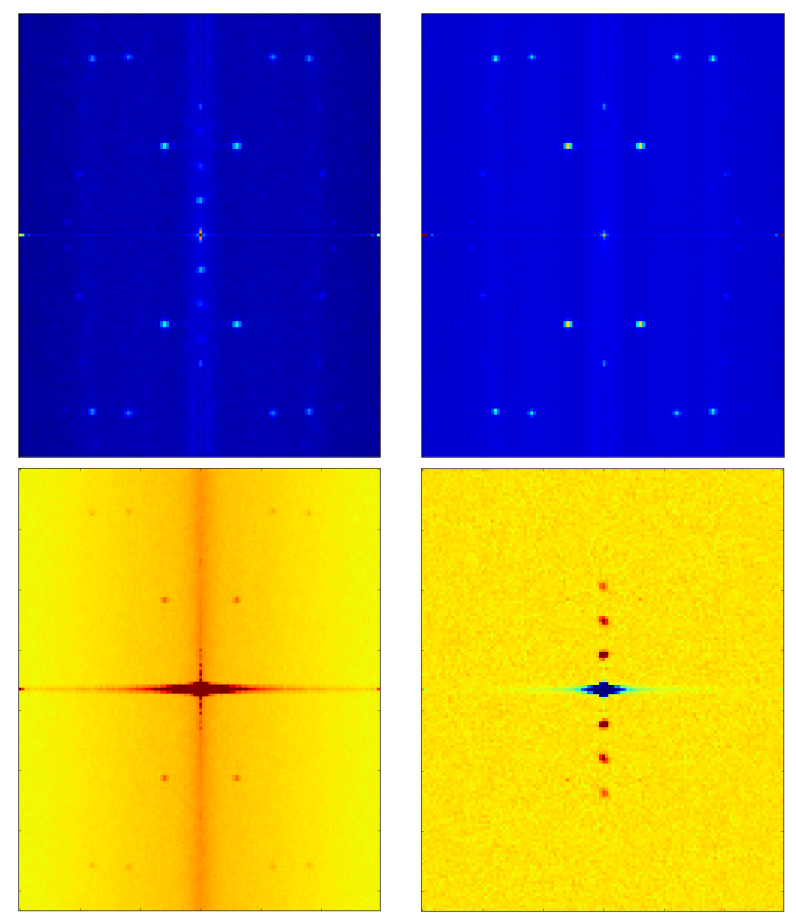

Fig. A.2. Different quantities used to obtain an unbiased mean $P S_{0}$ of the fringe data (bottom-right panel) over the frames. The mean $P S_{\text {dark }}$ (upper-right panel) removes the contribution of the dark (artifacts and background structures) in the mean $P S_{1}$ of the fringe (upper-left panel) and the mean $P S_{\text {foreground }}$ of the foreground data (bottom-left panel). The mean $P S_{\text {foreground }}$ removes the photon noise contribution and other background structures.

contribution of the photon bias structures in the PS of the fringe data (Garcia et al. 2016).

Step 5: We apply the photometric sorting on beams $i$ and $j$ on the preprocessed frames of the science data and compute the raw PS ( $\mathrm{PS}_{1}$, Fig. A.2, upper-left) for the fringes of baseline $i-j$. This PS is corrected following :

$\mathrm{PS}_{0}=\mathrm{PS}_{1}-\mathrm{PS}_{\text {dark }}-N_{1} / N_{\text {foreground }}\left(\mathrm{PS}_{\text {foreground }}-\mathrm{PS}_{\text {dark }}\right)$ 
where $\mathrm{PS}_{\mathrm{dark}}, \mathrm{PS}_{\text {foreground }}$ are respectively the PS of the dark and the PS of the foreground data (see Fig. A.2, resp. upper-right and bottom-left), and $N_{1}, N_{\text {foreground }}$ are respectively the number of photons in $\mathrm{PS}_{1}$ and $\mathrm{PS}_{\text {foreground. Despite this correction, a }}$ residual uniform bias, which integration represents around $10 \%$ of the energy of the fringe-peak, is found in $\mathrm{PS}_{0}$ (Fig. A.2, bottom-right). We identified it to the excess noise described in Robbins \& Hadwen (2003) and Hirsch et al. (2013) and decided to remove it after an estimation over an area with no data signal.

The position of the fringe signals in the final PS is used to estimate the optical path difference (OPD) following the method described by Koechlin et al. (1996). This quantity is first used to reject, by a method of sigma clipping, the measurements showing important fringe drifts or loss of fringe tracking. The temporal sampling of the OPD that can be reached depends on the $\mathrm{S} / \mathrm{N}$. The squared visibilities are then corrected from the nonzero OPD.

Step 6: The photometric sorting is applied on all photometric channels for the selection of frames used for the closure phase computation. As presented in Martinod et al. (2016), the closure phase estimation is based on the bispectrum of the preprocessed frames, $B_{1, u, v}$. It is unbiased following Eq. (A.3):

$B_{0, u, v}=B_{1, u, v}-\gamma\left(\left|C_{u}\right|^{2}+\left|C_{v}\right|^{2}+\left|C_{u+v}\right|^{2}\right)+\beta N$,

where $u$ and $v$ are respectively the spatial frequencies of the first and the second baseline. The term $\left|C_{u}\right|^{2}$ is the PS for the fringe at position $u$ and $N$ is the average photon counts in the interferogram. The $\gamma$ and $\beta$ coefficients allow to minimize the variance of the noise in the bispectrum area surrounding the region of interest.

\section{Appendix B: Expression of the shape factor}

The shape factor of the fringe-peak $\widetilde{\mathrm{Gab}}_{i j}$ is linked to the product of the beam pattern $\mathrm{Gab}_{i j}$ by the discrete Parseval's identity (because the PS of the interferogram is computed with a discrete FT)

$\widetilde{\mathrm{Gab}_{i j}}=N_{\text {pix }} \mathrm{Gab}_{i j}$

where $N_{\text {pix }}$ is the number of pixels in the interferogram.

In order to determine $\mathrm{Gab}_{i j}$, we assumed that the beam patterns are identical (same position, same full width at half maximum, same amplitude) and that the spectrum is flat with an intensity equal to 1 . The width of one spectral channel is fixed to 1 pixel. Consequently, $N_{\text {pix }}$ is defined by

$N_{\text {pix }}=\Delta \alpha \times N_{\lambda}$

where $\Delta \alpha$ and $N_{\lambda}$ are respectively the width of the interferogram along the spatial axis (in pixel) and the number of pixels along the spectral axis of the interferogram.

The beam pattern is defined by

$G(\lambda, \alpha)=A \mathrm{e}^{-\frac{\left(\alpha-\alpha_{0}\right)^{2}}{2 \sigma^{2}}}$,

with $\alpha_{0}$ and $\sigma$ the position and the characteristic dimension of the Gaussian function.

The interferogram is cut to $\alpha_{0} \pm 3 \sigma$ in the spatial direction so its width $\Delta \alpha=6 \sigma$.

The total flux in the Gaussian pattern is normalized to 1 in the interferometric channel

$\int_{\Delta \lambda} \int_{\Delta \alpha} G(\lambda, \alpha) \mathrm{d} \alpha \mathrm{d} \lambda=1$
From this equation, we can derive the expression of $A$

$A=\frac{1}{N_{\lambda} \sigma \sqrt{2 \pi}}$

The product of beam patterns $i$ and $j$ is

$\mathrm{Gab}_{i j}=\int_{\Delta \lambda} \int_{\Delta \alpha} G_{i}(\lambda, \alpha) G_{j}(\lambda, \alpha) \mathrm{d} \alpha \mathrm{d} \lambda$.

By combining Eqs. (B.3) and (B.5), we obtain

$\mathrm{Gab}_{i j}=N_{\lambda}\left(\frac{1}{N_{\lambda} \sigma \sqrt{2 \pi}}\right)^{2} \int_{\Delta \alpha} \mathrm{e}^{-\frac{\left(\alpha-\alpha_{0}\right)^{2}}{\sigma^{2}}} \mathrm{~d} \alpha$.

Since

$\int_{\Delta \alpha} \mathrm{e}^{-\frac{\left(\alpha-\alpha_{0}\right)^{2}}{\sigma^{2}}} \mathrm{~d} \alpha=\sqrt{2 \pi} \frac{\sigma}{\sqrt{2}}$

By combining Eqs. (B.7) and (B.8), we deduce the last expression of $\mathrm{Gab}_{i j}$ :

$\mathrm{Gab}_{i j}=\frac{1}{2 \sqrt{\pi} \frac{N_{\mathrm{pix}}}{6}}$

Finally, the expression of the shape factor is

$\widetilde{\mathrm{Gab}_{i j}}=\frac{3}{\sqrt{\pi}}$

\section{Appendix C: Demonstration of the photon contribution to the variance of the closure phase}

Tatulli \& Chelli (2005) give the expression of variance of closure phase in the photon noise regime by considering the photon noise and the Strehl ratio fluctuations. In this paper, we have neglected the Strehl ratio fluctuations. The photon noise contribution to the variance of the closure phase is given by Tatulli \& Chelli (2005):

$$
\begin{aligned}
& \sigma_{\mathrm{phot}}^{2}=\frac{N_{\text {tel }}^{3}}{2\left|V_{12} V_{23} V_{13}\right|^{2}}\left(\frac{N_{\text {tel }}}{N_{\mathrm{ph}}}\right)^{3}-\frac{1}{\left|V_{12} V_{23} V_{13}\right|}\left(\frac{N_{\text {tel }}}{N_{\mathrm{ph}}}\right)^{3} \\
&+\frac{\left.\left.N_{\mathrm{tel}}^{2}|| V_{12}\right|^{2}+\left|V_{23}\right|^{2}+\left|V_{13}\right|^{2}\right)}{2\left|V_{12} V_{23} V_{13}\right|^{2}}\left(\frac{N_{\mathrm{tel}}}{N_{\mathrm{ph}}}\right)^{2} \\
&-\frac{\left(\left|V_{12}\right|^{4}+\left|V_{23}\right|^{4}+\left|V_{13}\right|^{4}\right)}{\left|V_{12} V_{23} V_{13}\right|^{2}}\left(\frac{N_{\mathrm{tel}}}{N_{\mathrm{ph}}}\right)^{2} \\
&+\frac{\left(\left|V_{12}\right|^{2}\left|V_{23}\right|^{2}+\left|V_{23}\right|^{2}\left|V_{13}\right|^{2}+\left|V_{12}\right|^{2}\left|V_{13}\right|^{2}\right)}{\left|V_{12} V_{23} V_{13}\right|^{2}}\left(\frac{N_{\mathrm{tel}}}{N_{\mathrm{ph}}}\right)^{2} \\
&-\frac{\left|V_{12}\right|^{2}\left|V_{23}\right|^{2}+\left|V_{23}\right|^{2}\left|V_{13}\right|^{2}+\left|V_{12}\right|^{2}\left|V_{13}\right|^{2}}{2\left|V_{12} V_{23} V_{13}\right|^{2}}\left(\frac{N_{\mathrm{tel}}^{2}}{N_{\mathrm{ph}}}\right) \\
& 2\left|V_{12} V_{23} V_{13}\right|\left(\left|V_{12}\right|^{2}+\left|V_{23}\right|^{2}+\left|V_{13}\right|^{2}\right) \\
&\left.V_{23} V_{13}\right|^{2}
\end{aligned}
$$

with $\left|V_{i j}\right|$ the modulus of the visibility of beams $i$ and $j$.

The boxed terms of this equation depend on the coherent flux $C_{i j}=N_{\mathrm{ph}} V_{i j}$ and the photon noise variance $\sigma_{\mathrm{p}}^{2}=N_{\mathrm{ph}}$. It seems 
$N_{\text {ph }}$ has been simplified between them in Tatulli \& Chelli (2005). To use this equation for an EMCCD, we firstly explicit $\sigma_{\mathrm{p}}^{2}$ :

$$
\begin{aligned}
\sigma_{\mathrm{phot}}^{2} & =\frac{N_{\mathrm{tel}}^{3}}{2\left|V_{12} V_{23} V_{13}\right|^{2}}\left(\frac{N_{\mathrm{tel}}^{3}}{N_{\mathrm{ph}}^{6}}\right)\left(\sigma_{\mathrm{p}}^{2}\right)^{3}-\frac{1}{\left|V_{12} V_{23} V_{13}\right|}\left(\frac{N_{\mathrm{tel}}}{N_{\mathrm{ph}}}\right)^{3} \\
& +\frac{N_{\mathrm{tel}}^{2}\left(\left|V_{12}\right|^{2}+\left|V_{23}\right|^{2}+\left|V_{13}\right|^{2}\right)}{2\left|V_{12} V_{23} V_{13}\right|^{2}}\left(\frac{N_{\mathrm{tel}}^{2}}{N_{\mathrm{ph}}^{4}}\right)\left(\sigma_{\mathrm{p}}^{2}\right)^{2} \\
& -\frac{\left|V_{12}\right|^{4}+\left|V_{23}\right|^{4}+\left|V_{13}\right|^{4}}{\left|V_{12} V_{23} V_{13}\right|^{2}}\left(\frac{N_{\mathrm{tel}}}{N_{\mathrm{ph}}}\right)^{2} \\
& +\frac{\left|V_{12}\right|^{2}\left|V_{23}\right|^{2}+\left|V_{23}\right|^{2}\left|V_{13}\right|^{2}+\left|V_{12}\right|^{2}\left|V_{13}\right|^{2}}{\left|V_{12} V_{23} V_{13}\right|^{2}}\left(\frac{N_{\mathrm{tel}}}{N_{\mathrm{ph}}}\right)^{2} \\
& +\frac{\left|V_{12}\right|^{2}\left|V_{23}\right|^{2}+\left|V_{23}\right|^{2}\left|V_{13}\right|^{2}+\left|V_{12}\right|^{2}\left|V_{13}\right|^{2}}{2\left|V_{12} V_{23} V_{13}\right|^{2}}\left(\frac{N_{\mathrm{tel}}^{2}}{N_{\mathrm{ph}}^{2}}\right)\left(\sigma_{\mathrm{p}}^{2}\right) \\
& -\frac{2\left|V_{12} V_{23} V_{13}\right|\left(\left|V_{12}\right|^{2}+\left|V_{23}\right|^{2}+\left|V_{13}\right|^{2}\right)}{2\left|V_{12} V_{23} V_{13}\right|^{2}}\left(\frac{N_{\mathrm{tel}}}{N_{\mathrm{ph}}}\right) .
\end{aligned}
$$

Finally, we changed the variance of the photon noise $\sigma_{\mathrm{p}}^{2}$ into the modified one $\sigma_{\text {mod }}^{2}($ Sect. 2.2):

$\sigma_{\text {mod }}^{2}=F^{2}\left(N_{\mathrm{ph}}+N_{\mathrm{d}+\mathrm{c}}\right)$.

It allows us to obtain the expression for the error on closure phase for FRIEND (Sect. 2.3.2):

$$
\begin{aligned}
\sigma_{\mathrm{phot}}^{2} & =\frac{N_{\mathrm{tel}}^{3}}{2\left|V_{12} V_{23} V_{13}\right|^{2}}\left(\frac{N_{\mathrm{tel}}^{3}}{N_{\mathrm{ph}}^{6}}\right)\left(\sigma_{\mathrm{mod}}^{2}\right)^{3}-\frac{1}{\left|V_{12} V_{23} V_{13}\right|}\left(\frac{N_{\mathrm{tel}}}{N_{\mathrm{ph}}}\right)^{3} \\
& +\frac{N_{\mathrm{tel}}^{2}\left(\left|V_{12}\right|^{2}+\left|V_{23}\right|^{2}+\left|V_{13}\right|^{2}\right)}{2\left|V_{12} V_{23} V_{13}\right|^{2}}\left(\frac{N_{\mathrm{tel}}^{2}}{N_{\mathrm{ph}}^{4}}\right)\left(\sigma_{\mathrm{mod}}^{2}\right)^{2} \\
& -\frac{\left|V_{12}\right|^{4}+\left|V_{23}\right|^{4}+\left|V_{13}\right|^{4}}{2\left|V_{12} V_{23} V_{13}\right|^{2}}\left(\frac{N_{\mathrm{tel}}}{N_{\mathrm{ph}}}\right)^{2} \\
& +\frac{\left|V_{12}\right|^{2}\left|V_{23}\right|^{2}+\left|V_{23}\right|^{2}\left|V_{13}\right|^{2}+\left|V_{12}\right|^{2}\left|V_{13}\right|^{2}}{\left|V_{12} V_{23} V_{13}\right|^{2}}\left(\frac{N_{\mathrm{tel}}}{N_{\mathrm{ph}}}\right)^{2} \\
& +\frac{\left|V_{12}\right|^{2}\left|V_{23}\right|^{2}+\left|V_{23}\right|^{2}\left|V_{13}\right|^{2}+\left|V_{12}\right|^{2}\left|V_{13}\right|^{2}}{2\left|V_{12} V_{23} V_{13}\right|^{2}}\left(\frac{N_{\mathrm{tel}}^{2}}{N_{\mathrm{ph}}^{2}}\right)\left(\sigma_{\text {mod }}^{2}\right) \\
& -\frac{\left.\left.\left|V_{12} V_{23} V_{13}\right||| V_{12}\right|^{2}+\left|V_{23}\right|^{2}+\left|V_{13}\right|^{2}\right)}{\left|V_{12} V_{23} V_{13}\right|^{2}}\left(\frac{N_{\mathrm{tel}}}{N_{\mathrm{ph}}}\right) .
\end{aligned}
$$

where $\left|V_{i j}\right|$ is the modulus of the visibility of beams $i$ and $j$.

\section{Appendix D: Choice of the crystal used for the compensation of the birefringence}

We followed the work done by Lazareff et al. (2012) in order to choose the material for the compensation plates. Our instrument required a birefringence correction of the order of $5 \mu \mathrm{m}$ between two beams of the interferometer. Besides, the dispersion (versus wavelength) of the birefringence correction should be as small as possible in order to have a fringe contrast lost better than $1 \%$

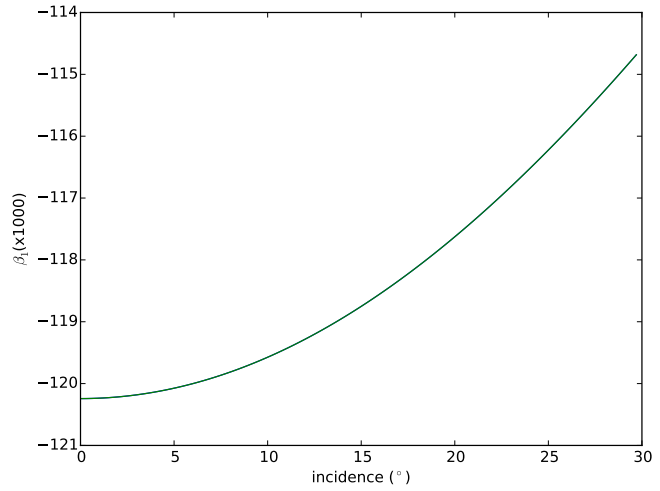

Fig. D.1. Effective birefringence versus incidence at $\lambda=690 \mathrm{~nm}$.

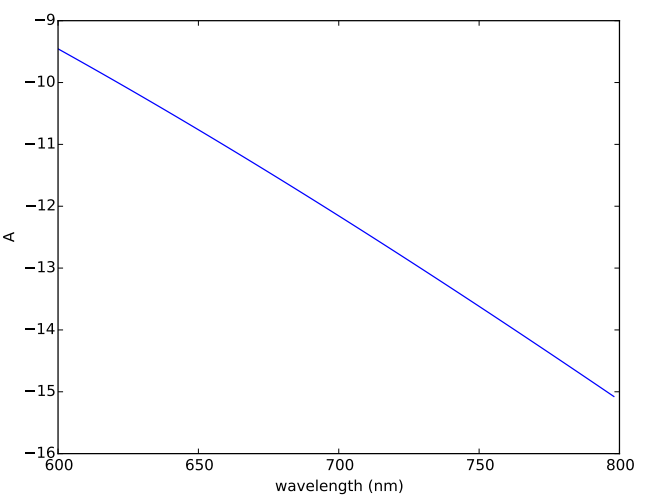

Fig. D.2. Figure of merit A with respect to wavelength.

over the whole spectral bandwidth of FRIEND $(\Delta \lambda=120 \mathrm{~nm}$ between $630 \mathrm{~nm}$ and $750 \mathrm{~nm}$ ).

We studied several uniaxial crystals. Finally we chose the high temperature phase $\mathrm{BBO}$, called $\alpha B B O$, which is a negative uniaxial crystal with a large birefringence over the broad transparent range from $189 \mathrm{~nm}$ to $3500 \mathrm{~nm}$. To check our specifications we computed two quantities: the effective birefringence $\beta_{\text {eff }}$ and the figure of merit $A$ (see Eq. (5) and Sect. 4 in Lazareff et al. 2012). Figure D.1 presents the behavior of $\beta_{\text {eff }}$ with respect to the incidence angle at $\lambda=690 \mathrm{~nm}$. And Fig. D. 2 presents the behavior of $A$ with respect to the wavelength.

Adopting an incidence angle excursion from $0^{\circ}$ to $30^{\circ}$, Fig. D.1 shows that we can achieve an effective birefringence excursion of $\approx 5 \times 10^{-3}$. So with a plate thickness of $2 \mathrm{~mm}$, the system provides a peak-to-peak correction of $10 \mu \mathrm{m}$ path length. This correction is in perfect agreement with our specification $(5 \mu \mathrm{m})$ since, in our system, one of the three compensation plates is fixed.

Adopting a maximal birefringence of $5 \mu \mathrm{m}$ at $690 \mathrm{~nm}$ corresponding to $n_{\mathrm{f}}$ is equal to seven fringes, our compensation introduce at the band edges a differential birefringence of

$\delta n_{\text {edge }} \approx n_{\mathrm{f}} \frac{1}{|A|} \frac{\Delta \lambda}{2 \lambda} \approx 0.05$

where $|A| \approx 12$ at $\lambda=690 \mathrm{~nm}$ (see Fig. D.2). This differential birefringence of 0.05 fringes induces a $1 \%$ lost of contrast at the band edges which is also in good agreement with our specifications. 